Upscaling of a tri-phase phase-field model for precipitation in porous media Non Peer-reviewed author version

Redeker, Magnus; Rohde, Christian \& POP, Sorin (2016) Upscaling of a tri-phase phase-field model for precipitation in porous media. In: IMA JOURNAL OF APPLIED MATHEMATICS, 81 (5), p. 898-939.

DOI: 10.1093/imamat/hxw023

Handle: http://hdl.handle.net/1942/22552 


\title{
Upscaling of a Tri-Phase Phase-Field Model for Precipitation in Porous Media
}

\author{
MAGNus REDEKER \\ Universität Stuttgart, Institut für Angewandte Analysis und Numerische Simulation, \\ Pfaffenwaldring 57, D-70569 Stuttgart, Germany, \\ magnus.redeker@mathematik.uni-stuttgart.de \\ AND \\ IULIU SORIN POP \\ CASA, Department of Mathematics and Computer Science, Eindhoven University of \\ Technology, PO Box 513, 5600 MB Eindhoven, The Netherlands, \\ i.popdtue.nl \\ AND \\ CHRISTIAN ROHDE \\ Universität Stuttgart, Institut für Angewandte Analysis und Numerische Simulation, \\ Pfaffenwaldring 57, D-70569 Stuttgart, Germany, \\ christian.rohde@mathematik.uni-stuttgart.de
}

[7 October 2014]

\begin{abstract}
We consider a porous medium with a pore space that is completely filled by three different phases: two immiscible fluids (say water and oil) and a solid phase. One fluid phase contains dissolved ions, which can precipitate at the pore boundary to form the solid phase. The reverse process of dissolution, is also possible. Consequently, the solid phase changes in time; its variation is not known a-priori. The second fluid contains no solute and has no interaction with the solid phase.

Starting from a standard sharp interface model for the pore-scale dynamics we develop a diffuse interface approach that accounts for the time-dependent spatial distribution of the three species and the overall concentration of the solute. Basic analytical results for this model are presented, including the well posedness of the phase field component of the model. Next we apply matched asymptotic techniques to show that the diffuse interface model converges to the sharp interface one. Further, homogenization is applied to derive a new two-scale model that is valid at the Darcy scale. This leads to a parabolic reaction-diffusion system in a medium with variable, concentration dependent porosity. The diffuse interface approach allows describing the variation in the porosity as phase field type equations at the pore-scale.

The last part of the paper presents an efficient numerical scheme to approximate the solution of the twoscale model. This scheme has as starting point the algorithm in [M. Redeker and C. Eck. A fast and accurate adaptive solution strategy for two-scale models with continuous inter-scale dependencies. J. Comput. Phys., 240:268-283, 2013]. After some test cases validating the method, we finally present computations for several realistic scenarios. The results demonstrate the interdependence of the change of the pore structure due to precepitaion/dissolution and the evolution of the Darcy scale concentration of the dissolved particles in the one fluid.
\end{abstract}

Keywords: Multiphase Porous Media, Phase Field Models, Homogenization, Heterogeneous Multiscale Method

\section{Introduction}

We are concerned with the multi-scale modeling for a precipitation/dissolution process in a porous medium that involves three phases. Precisely, the medium's pore-space is occupied by two immiscible, incompressible fluids (say water and oil) and by a crystalline solid or precipitate like salt, which can only dissolve in one phase (water). This different behaviour is the motivating background for having two fluidic phases. In this context, three interfaces are encountered. If the precipitate is surrounded by water on one part of its boundary and by oil on the other, then it can 
only dissolve and precipitate through the water-precipitate interface, which is moved by dissolution and precipitation, and by curvature effects. The oil-precipitate interface instead is assumed to be chemically inactive and only driven by curvature. Finally, the third interface separates oil and water. Its dynamics is given by curvature effects; advection can be added too. The major unknowns in this setting are the solute concentration in the water and the position of the interfaces. Note that the concentration of species forming the precipitate is zero in the oil phase and fixed (more precise, one, by scaling) in the precipitate phase. However, due to precipitation, dissolution and transport, it is variable in the water phase. Finally, the last phase is an immobile phase attached to the pore walls/grains. It is the result of precipitation and dissolution, therefore it is called "precipitate". Assuming that the precipitate layer has a non-negligible size when compared to the pore size, precipitation and dissolution are changing the pore space available to fluids. This means that the equations for the fluid phases are defined in time-dependent domains. Their evolution is not known a-priori and depends on the concentration of the solute in the water phase. From this point of view, the corresponding mathematical models are involving free boundaries at the pore scale. At the Darcy scale, this results in a model with variable, concentration dependent porosity. The extreme situation is when the growth of the precipitate layer leads to an obstruction in the pore space, the so-called pore clogging effect. In mathematical terms, this is similar to a topological change of the pore space.

Various approaches are possible for modelling processes involving free boundaries at the pore scale. The general difficulty is describing their evolution. Strictly related to dissolution and precipitation, we refer to Noorden \& Pop (2008) for the analysis of a model in the simple, one dimensional case, where only two phases (water and precipitate) are present. In this case, the model can be transformed to one posed in a fixed domain. This approach can be extended for simple geometries, like a strip. This is the framework in Noorden (2009b), where the time scales for fluid flow and for diffusion are assumed in balance, and in Kumar et al. (2011) for convection-dominated regimes (also see Kumar et al. (2013)). In these cases, the interface between the fluid and the precipitate is described as a function of time and space.

The micro-scale interfaces considered in Peter (2009) have a prescribed evolution, allowing a re-mapping to a reference configuration. In this context, rigorous homogenization results are obtained. Formal homogenization for a reactive transport model posed in a complex, micro-scale domain with a-priori unknown evolution is performed in Meier (2008). Again, the situation allows re-mapping to a reference configuration. The situation analyzed in Muntean \& Noorden (2013); Noorden \& Muntean (2011) is somewhat similar. There the evolving interfaces are replaced by a locally periodic medium, with solid phases having fixed, but different distribution from one reference cell to another.

A different approach, proposed in Noorden (2009a), works for more general geometries: the interface between the precipitate and the fluid phase is the zero level set of a function being, say, positive in the domain occupied by the precipitate phase, and negative in the fluid phase. Related are models encountered e.g. for drug release from collagen matrices (see Ray et al. (2013)). A third approach, based on phase fields, is adopted in our work. From modelling point of view, it builds on the model proposed in Noorden \& Eck (2011), where a domain occupied by two phases (fluid and precipitate) is considered. Similar to the situation described above, the interface separating the two phases is moving by chemical processes and by curvature. Related are also the works Eck \& Emmerich (2009); Eck et al. (2013), dealing with the upscaling of a phase field model for liquid-phase epitaxy.

It is important mentioning that in all papers mentioned before only two phases are considered. Viewed in the context of porous media, these models account for the changes in the pore aperture or geometry, but assume that the medium is fully saturated by one fluid phase. The present work extends this framework by including a second fluid phase that is not miscible with the first one (like oil, or even air next to the water) and behaving differently with respect to the solute and the precipitate. In other words, we consider two fluid phases in the porous medium, or unsaturated media. Having this as starting point, here an upscaled (Darcy scale) model is being derived.

This paper is organized as follows. Firstly in Section 2, we develop a tri-phasic pore-scale model that relies on the sharp-interface ansatz. It models the evolution of the concentration of the dissolved precipitate in water and the evolution of the interfaces: water-oil, water-precipitate, oil-precipitate. The interfaces' evolutions are determined by kinetic conditions. In particular, the precipitation and dissolution process at the water-precipitate interface, while Stefan conditions at the water-oil and at the water-precipitate interface ensure the conservation of mass of the dissolved precipitate in water. This ansatz generalizes the models from Noorden \& Pop (2008), or Noorden \& Eck (2011), which are only concerned with a water and a precipitate phase. In the next step we suggest a phase field approximation for the tri-phasic sharp-interface model. For the general framework in phase field modeling we refer to Caginalp (1986) and to Boyer \& Lapuerta (2006); Boyer et al. (2010) for the handling of a three phase setting. Our ansatz describes the evolution of three phase fields and the evolution of the concentration of the dissolved particles in the water. Besides precipitation and dissolution, the evolution of the concentration is also influenced by diffusion. Motivated by the 
Diffuse Domain Approximation (DDA) method introduced in Li et al. (2009), each phase-field is treated as a smooth approximation to the characteristic function - in the sharp-interface sense - of one of the three phases involved: water, oil and the precipitate. Within a small phase-transition region a phase-field for a substance varies smoothly from the value one inside the associated region to zero inside the regions occupied by the two other substances. We provide basic well-posedness results for this model. In particular a formal asymptotic analysis shows that the solution of the phase-field model tends in the sharp-interface limit to the solution of the sharp-interface model.

Secondly, considering in Section 3 a situation with periodically distributed grains surrounded by the three phases with a period of scale $\varepsilon>0$, the application of a formal periodic homogenization technique to the pore-scale phase field model yields in the limit for $\varepsilon \rightarrow 0$ a two-scale model. It is suitable for settings where the dissolved particles in the water diffuse on the Darcy-scale. It consists of a macroscopic homogenized diffusion equation for the concentration of particles in the water, and, for each point of the macroscopic domain, of local cell problems for the evolution of the three phases. The variables on the different scales depend continuously on each other: the growth of the microscopic solid part (grain and precipitate) is influenced by the macroscopic concentration of the dissolved particles in the water, and vice versa the change of the pore structure influences the evolution of the concentration.

Thirdly, we propose an efficient numerical multi-scale algorithm to solve the upscaled model. The core of the numerical method is the adaptive strategy that has been introduced in Redeker \& Eck (2013) in the context of a completely different solidification process. Section 4 presents then numerical results for the upscaled model in two spatial dimensions that document the interdependence of the concentration of the dissolved particles on the Darcyscale and the change of the pore structure. In particular, local clogging effects are documented.

To conclude the introduction, we mention that the reason for considering a phase-field model instead of the original sharp-interface model is its simplicity from different point of views: mathematical analysis, upscaling and numerical discretization. At the expense of three additional unknowns and equations, the phase field model is defined in the entire domain, and no free boundaries need to be considered as in the sharp interface case. The developed porescale phase-field model bears a slight resemblance to a model introduced in Noorden \& Eck (2011): both models utilize a pore-scale phase-field formulation describing a precipitation process in a porous medium. However, there are considerable differences. Firstly, the Noorden \& Eck (2011)-model considers only two substances, the precipitate and one liquid containing dissolved particles (solute). Secondly, the complicated structure of the evolution equation for the solute concentration in Noorden \& Eck (2011) makes homogenization a difficult task, whereas here it is quite straightforward. Thirdly, the present approach can be easily extended to more than three substances, which is, again, nontrivial for the model in Noorden \& Eck (2011). Some hints in this sense are given in Section 5.

\section{The pore-scale model}

This section briefly discusses the sharp-interface model that is approximated by the proposed pore-scale phase-field model. Next, it develops the pore-scale phase-field model and shows that the model describes a perfect mixture almost everywhere in the time-space cylinder - a perfect mixture in the sense that the sum of the three phase-fields equals one and that each phase-field is greater or equal to zero. Finally, this section shows that the solution of the phase-field model approximates in the limit for the width of the phase transition regions tending to zero the solution of the sharp-interface model.

\subsection{The sharp-interface model}

Consider a time-interval $J(T):=(0, T], T>0$, with $\overline{J(T)}=[0, T]$, and a spatial domain $\Omega \subset \mathbb{R}^{d}, d \in\{2,3\}$, with a boundary $\Gamma_{\Omega}=\partial \Omega . \Omega$ is subdivided into the pore-space $\mathscr{P}$, a grain $\mathscr{G}$ and the boundary $\Gamma_{\mathscr{G}}$ of the grain, that is $\Omega=\mathscr{P} \cup \mathscr{G} \cup \Gamma_{\mathscr{G}}$. The boundary $\Gamma_{\mathscr{P}}$ of the pore-space $\mathscr{P}$ equals $\Gamma_{\Omega} \cup \Gamma_{\mathscr{G}}$. Let $n_{\mathscr{P}}$ be the outer-normal to $\Gamma_{\mathscr{P}}$. The pore-space $\mathscr{P}$ is occupied by three substances $P_{i}, i \in I:=\{1,2,3\}$ : two immiscible, incompressible fluids $P_{1}$ (e.g. water) and $P_{2}$ (e.g. oil) and the precipitate $P_{3}$. The substances occupy three disjoint time-dependent a priori unknown regions $P_{1}^{s}(t), P_{2}^{s}(t)$ and $P_{3}^{s}(t)$. These regions are separated by the moving interfaces $\Gamma_{i j}^{s}(t)=\partial P_{i}^{s}(t) \cap \partial P_{j}^{s}(t), i, j \in I$ and $j>i$, where the normal $n_{i j}$ to $\Gamma_{i j}^{s}(t)$ points into the domain $P_{j}^{s}(t)$. Therefore,

$$
\mathscr{P}=P_{1}^{s}(t) \cup P_{2}^{s}(t) \cup P_{3}^{s}(t) \cup \Gamma_{12}^{s}(t) \cup \Gamma_{13}^{S}(t) \cup \Gamma_{23}^{s}(t) .
$$

Throughout this article three initial configurations $\mathscr{I}=\left\{\mathscr{I}_{0}, \mathscr{I}_{1}, \mathscr{I}_{2}\right\}$ of the three phasees are considered. In each configuration, as depicted in Figure 2.1, the precipitate $P_{3}$ surrounds the grain which is located in the center of the pore-space $\mathscr{P}$. In initial configuration $\mathscr{I}_{0}$ the fluid $P_{2}$ is not existent, that is the remainder of the non-precipitate porespace $\mathscr{P} \backslash P_{3}^{s}(0)$ is fully occupied by the fluid $P_{1}$. On contrary, in initial configuration $\mathscr{I}_{1}$ the fluid $P_{2}$ is existent. The 


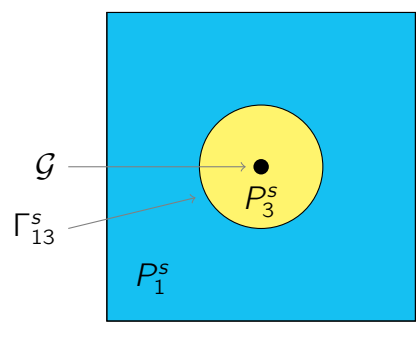

(a) Initial configuration $\mathscr{I}_{0}$.

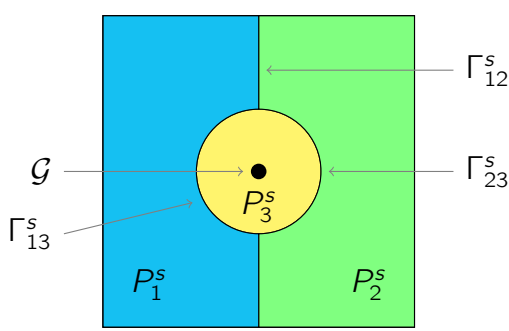

(b) Initial configuration $\mathscr{I}_{1}$.

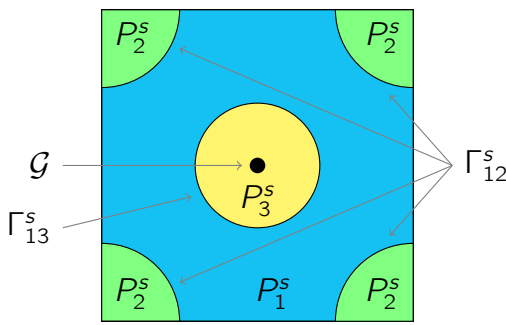

(c) Initial configuration $\mathscr{I}_{2}$.

FIG. 2.1: The three considered initial configutations $\mathscr{I}_{0}, \mathscr{I}_{1}$ and $\mathscr{I}_{2}$ on the pore-scale. The domain $\Omega$ consists of the pore-space $\mathscr{P}$, the grain $\mathscr{G}$, which is located in the center of $\Omega$, and the grain-boundary $\Gamma_{\mathscr{G}}=\partial \mathscr{G}$. The boundary $\Gamma_{\mathscr{P}}$ of the pore-space $\mathscr{P}$ satisfies $\Gamma_{\mathscr{P}}=\Gamma_{\Omega} \cup \Gamma_{\mathscr{G}}$, where $\Gamma_{\Omega}=\partial \Omega$ denotes the outer-boundary of $\Omega$. The pore-space is occupied by the two immiscible and incompressible fluids $P_{1}$ in $P_{1}^{s}$ and $P_{2}$ in $P_{2}^{s}$, the precipitate $P_{3}$ in $P_{3}^{s}$, and their interfaces $\Gamma_{12}^{s}, \Gamma_{13}^{s}$ and $\Gamma_{23}^{s}$. In each configuration, the precipitate $P_{3}$ is attached to the boundary $\Gamma_{\mathscr{G}}$ of the grain $\mathscr{G}$. In initial configuration $\mathscr{I}_{0}$ the fluid $P_{2}$ is not existent. Consequently, the fluid $P_{1}$ fully occupies the remainder of the non-precipitate pore-space. On contrary, in initial configuration $\mathscr{I}_{1}$ the fluid $P_{2}$ is existent. It occupies the right part and the fluid $P_{1}$ the left part of the non-precipitate pore-space. Finally, in initial configuration $\mathscr{I}_{2}$ the fluid $P_{2}$ is located in the corners of the microscopic domain $Y$, that is $P_{2}^{s}$ is spherical in case of periodic boundary conditions.

non-precipitate pore-space is occupied by $P_{1}$ on the left and by $P_{2}$ on the right of the precipitate. Finally, in initial configuration $\mathscr{I}_{2}$ the fluid $P_{2}$ is located in the corners of the microscopic domain $Y$. In case of periodic boundary conditions $P_{2}^{s}(0)$ is spherical.

The fluid $P_{1}$ in $P_{1}^{s}(t)$ contains dissolved particles that can precipitate on the $P_{1}$-part $\partial P_{1}^{s}(t) \cap \Gamma_{\mathscr{G}}$ of the grainboundary $\Gamma_{\mathscr{G}}$ as well as on the boundary $\Gamma_{13}^{s}(t)$. The domains $P_{2}^{s}(t)$ resp. $P_{3}^{s}(t)$ are occupied by the fluid $P_{2}$ resp. $P_{3}$. The concentration $u$ of particles is fixed in these two regions. By assumption, $P_{2}$ does not contain particles, hence $u=0$ in $P_{2}^{s}(t)$. Furthermore, let $\rho>0$ be the fixed concentration of particles in the precipitate region $P_{3}^{s}(t)$. The following sharp-interface model accounts for the diffusion process of the dissolved particles' concentration $u$ in $P_{1}^{s}(t)$, for mass conservation at the moving interfaces and for the precipitation process.

Problem 2.1 Given the constant diffusion coefficient $D>0$, the normal velocity $v_{12}^{n}$ of the moving interface $\Gamma_{12}^{s}(t)$ and the normal velocity $v_{13}^{n}$ of $\Gamma_{13}^{s}(t)$, the concentration $\rho>0$ of the components in $P_{3}$, and the initial condition $u_{0}: P_{1}^{s}(0) \rightarrow \mathbb{R}$, find the concentration $u$ satisfying

$$
\begin{array}{ll}
\partial_{t} u-D \Delta u=0 & \text { in } J(T) \times P_{1}^{s}(t), \\
-D \nabla u \cdot n=0 & \text { on } J(T) \times\left(\partial P_{1}^{s}(t) \cap \Gamma_{\mathscr{P}}\right), \\
-D \nabla u \cdot n_{12}=v_{12}^{n} u & \text { on } J(T) \times \Gamma_{12}^{s}(t), \\
-D \nabla u \cdot n_{13}=v_{13}^{n}(u-\rho) & \text { on } J(T) \times \Gamma_{13}^{s}(t), \\
u(0, x)=u_{0}(x) & \text { in } P_{1}^{s}(0) .
\end{array}
$$

Since $u=0$ in $P_{2}^{s}(t)$ and $u=\rho$ in $P_{3}^{s}(t)$, the equations $\left(2.2_{3,4}\right)$ are in fact Rankine-Hugoniot conditions at the moving interfaces of $P_{1}^{S}(t)$, ensuring the conservation of mass. Further, the normal velocities there are not known a-priori, but given by the following kinetic conditions

$$
\begin{aligned}
& v_{12}^{n}=-\sigma \kappa_{12} \text { on } J(T) \times \Gamma_{12}^{s}(t), \\
& v_{13}^{n}=-\sigma \kappa_{13}-f(u) \text { on } J(T) \times \Gamma_{13}^{s}(t), \\
& v_{23}^{n}=-\sigma \kappa_{23} \text { on } J(T) \times \Gamma_{23}^{s}(t),
\end{aligned}
$$

with the constant surface tension $\sigma>0$ and the mean curvatures $\kappa_{i j}$ of the interfaces $\Gamma_{i j}^{S}(t), i, j \in I, j>i$. In order to 


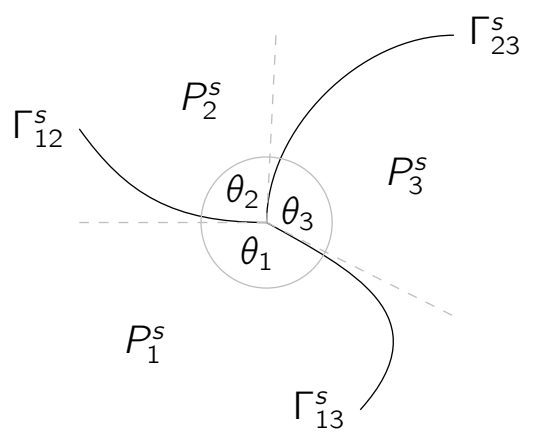

FIG. 2.2: The three phases and their interfaces.

ensure that the interfaces do not leave the pore-space, the following conditions are imposed

$$
\begin{aligned}
& v_{12}^{n}=\min \left(0,-\sigma \kappa_{12}\right) \text { on } J(T) \times\left(\Gamma_{12}^{s}(t) \cap \Gamma_{\mathscr{P}}\right), \\
& v_{13}^{n}=\min \left(0,-\sigma \kappa_{13}-f(u)\right) \text { on } J(T) \times\left(\Gamma_{13}^{s}(t) \cap \Gamma_{\mathscr{P}}\right), \\
& v_{23}^{n}=\min \left(0,-\sigma \kappa_{23}\right) \text { on } J(T) \times\left(\Gamma_{23}^{s}(t) \cap \Gamma_{\mathscr{P}}\right),
\end{aligned}
$$

where $v_{i j}^{n}$ denotes in this case the velocity of the moving interface $\Gamma_{i j}^{s}(t)$ into the outer-normal direction $n \mathscr{P}$. The initial configuration $P_{1}^{s}(0), P_{2}^{s}(0)$ and $P_{3}^{s}(0)$ is given.

The rate function $f(u)$ drives the precipitation process. For the ease of presentation we use here a simple affine linear function

$$
f(u)=c_{u, 0}\left(u-c_{u, 1}\right),
$$

with given constant rate $c_{u, 0}>0$ and constant threshold $0<c_{u, 1}<\rho$ separating precipitation and dissolution. If the concentration is larger than the threshold, then dissolved particles precipitate and the precipitate phase expands. On the other hand, the precipitate phase recedes (due to dissolution) if the concentration is below the threshold. More involved models are discussed in Knabner et al. (1995) and Noorden \& Pop (2008).

REMARK 2.1 In general, the surface tension of a solid/precipitate is anisotropic because of the crystalline structure. For simplicity only constant isotropic tensions are considered. Hence, the numerical results do not show dendritic growth. Referring to Figure 2.2, where $\Theta_{1}, \Theta_{2}$ and $\Theta_{3}$ denote the angles between the interfaces, when considering two spatial dimensions Young's law gives

$$
\frac{\sin \left(\Theta_{1}\right)}{\sigma}=\frac{\sin \left(\Theta_{2}\right)}{\sigma}=\frac{\sin \left(\Theta_{3}\right)}{\sigma} .
$$

Note that the surface tension between any two phases is assumed the same, $\sigma$. This means that at any common junction point of the three interfaces $\Gamma_{12}^{s}(t), \Gamma_{13}^{s}(t)$ and $\Gamma_{23}^{s}(t)$, these meet at an angle of $120^{\circ}$.

REMARK 2.2 From the physical point of view, the boundary $\Gamma_{23}^{s}(t)$ separating the oil and the precipitate should be immobile, and the boundary $\Gamma_{13}^{s}(t)$ separating the water and the precipitate should only be affected by precipitation. However, this sharp-interface model is tailored to the phase-field model developed below, in the sense that its solution is approximated by the solution of the phase field model. Since the evolution of the diffuse interface in a phase-field model is affected by its curvature, the mean curvatures of the sharp-interfaces $\Gamma_{13}^{s}(t)$ and $\Gamma_{23}^{s}(t)$ are included here. Further details are presented in Section 2.3.

\subsection{The phase-field model}

The aim is to develop an upscaled model that allows for an efficient numerical solution. Because it is usually much easier to deal with phase-field instead of sharp-interface models when it comes to numerical simulations as well as upscaling, the sharp interface model of the previous subsection is approximated by a phase-field model. According to the three considered pure states $P_{1}, P_{2}$ and $P_{3}$, three phase-fields are introduced. For $i \in I$, the phase-field $\phi_{i}(t, x)$ is 


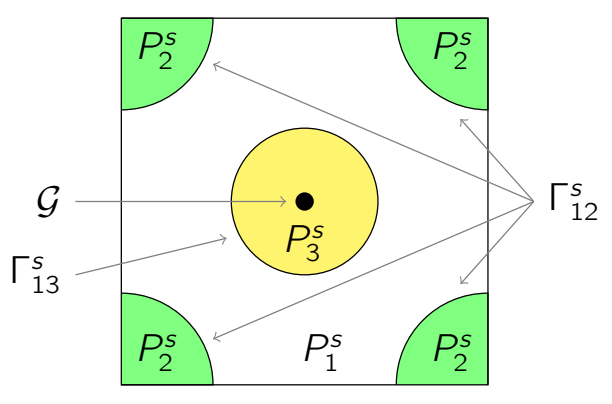

(a) Sharp interfaces.

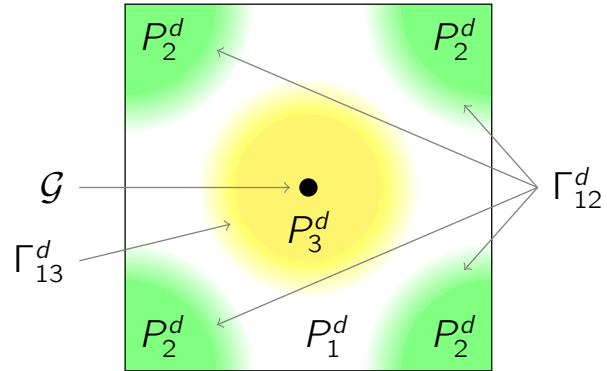

(b) Diffuse interfaces.

FIG. 2.3: In the diffuse interface setting the phase-field $\phi_{i} \in[0,1]$ smoothly approximates the characteristic function $\chi_{i} \in\{0,1\}$ of the sub-domain $P_{i}^{s}$ in the sharp interface setting. The sharp interfaces $\Gamma_{i j}^{s}$ are approximated by the diffuse interface regions $\Gamma_{i j}^{d}$, in which the phase-fields $\phi_{i}$ and $\phi_{j}$ vary smoothly between 0 and 1 .

treated as a smooth approximation to the characteristic function $\chi_{i}(t, x)$ of the sharp-interface domain $P_{i}^{s}(t)$. Figure 2.3 sketches the approximation.

At first, this subsection develops a system of three evolution equations for the three phase-fields. Thereafter, motivated by the Diffuse Domain Approximation (DDA) method in Li et al. (2009), the sharp-interface model's evolution equation in (2.2), which models the diffusion process for the concentration $u$ of particles in the fluid region $P_{1}^{s}(t)$, is transformed into an equation that is valid in the whole pore-space $\mathscr{P}$. Additional source terms approximate the Rankine-Hugoniot condition at the interfaces $\Gamma_{12}^{s}(t)$ and $\Gamma_{13}^{s}(t)$. Without going into details here, the phase-field $\phi_{1}$ plays a crucial role in the transformed equation due to the fact that it approximates the characteristic function of the fluid region $P_{1}^{s}(t)$.

For ease of the presentation the development of the phase-field model is subdivided into two parts. Firstly, the evolution equations for the phase-fields are developed for a given concentration. Secondly, for given phase-fields, the equation for the concentration is developed. Clearly, the two parts are coupled in the end.

\subsubsection{The evolution equations for the phase-fields $\Phi=\left(\phi_{1}, \phi_{2}, \phi_{3}\right)$}

For $i \in I$, the phase-field $\phi_{i}(t, x)$ is treated as a smooth approximation to the characteristic function $\chi_{i}(t, x)$ of the sharp-interface domain $P_{i}^{s}(t)$. Therefore, the phase fields must satisfy

$$
0 \leqslant \phi_{i} \leqslant 1 \text { and } \sum_{i \in I} \phi_{i}=1
$$

a.e. in $J(T) \times \mathscr{P}$. Consequently, the admissible states of $\Phi=\left(\phi_{1}, \phi_{2}, \phi_{3}\right)$ belong to

$$
\mathscr{S}:=\left\{\Phi=\left(\phi_{1}, \phi_{2}, \phi_{3}\right) \in[0,1]^{3} \mid \sum_{i \in I} \phi_{i}=1\right\} .
$$

The Allen-Cahn type equations developed in the following guarantee that if the initial state $\Phi(0, x) \in \mathscr{S}$ for a.e. $x \in \mathscr{P}$ then the solution $\Phi(t, x) \in \mathscr{S}$ for a.e. $(t, x) \in J(T) \times \mathscr{P}$. The evolution of the system is driven by the gradient of a free energy $U(\Phi)$ given below and, additionally, the diffuse interface region $\Gamma_{13}^{d}(t)$ separating the liquid domain $P_{1}^{d}(t)$ and the precipitate $P_{3}^{d}(t)$ is driven by the precipitation process.

The free energy $U(\Phi)$ is given by

$$
U(\Phi)=\int_{\mathscr{P}} 3 W(\Phi(t, x))+\frac{3}{8} \xi^{2} \sum_{i \in I}\left|\nabla \phi_{i}(t, x)\right|^{2} d x .
$$

The potential $W=W(\Phi)$,

$$
W(\Phi)=2 \sum_{i \in I} \phi_{i}^{2}\left(1-\phi_{i}\right)^{2},
$$

with the partial derivatives

$$
\partial_{\phi_{i}} W(\Phi)=4\left(\phi_{i}-3 \phi_{i}^{2}+2 \phi_{i}^{3}\right) \text { for } i \in I,
$$




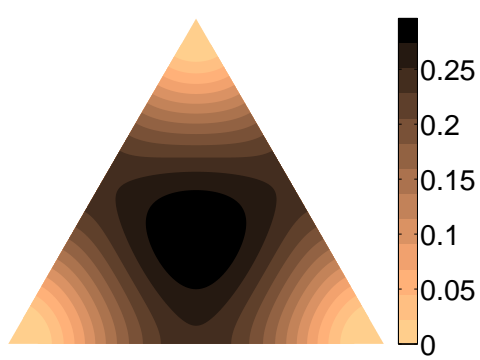

(a) $W(\Phi)=2 \sum_{i \in I} \phi_{i}^{2}\left(1-\phi_{i}\right)^{2}$.

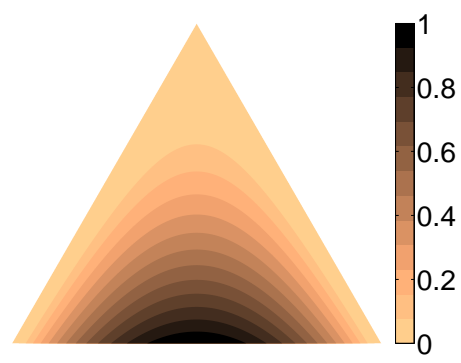

(b) $\gamma_{13}(\Phi)=4 \phi_{1} \phi_{3}$.

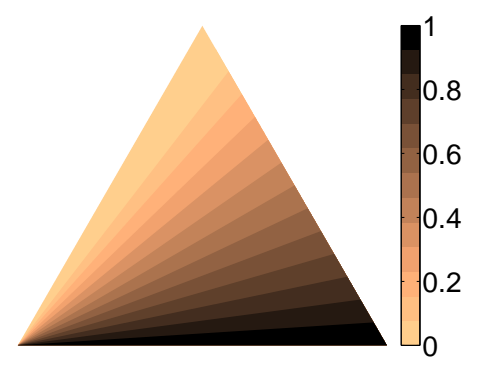

(c) $g_{\delta}(\Phi)=\left(\phi_{2}+\phi_{3}+\delta\right)^{-1} \phi_{3}$.

FIG. 2.4: Contour plots in barycentric coordinates for $\Phi=\left(\phi_{1}, \phi_{2}, \phi_{3}\right) \in \mathscr{S}$. The bottom left corner represents the pure fluid $\bar{P}_{1}^{d}$, that is $\Phi=(1,0,0)$, the top corner the fluid $\bar{P}_{2}^{d}$ and the bottom right corner the precipitate $\bar{P}_{3}^{d}$. The Figure (a) depicts the contour plot of the potential $W$, (b) of the indicator $\gamma_{13}$ of the diffuse interface $\bar{\Gamma}_{13}^{d}$ separating the fluid $\bar{P}_{1}^{d}$ and the precipitate $\bar{P}_{3}^{d}$, and (c) of the interpolation function $g_{\delta}$ with $\delta=1 e-6$.

is non-convex in $\mathscr{S}$ and minimal for the pure states $\bar{P}_{i}^{d}$,

$$
\bar{P}_{i}^{d}:=\left\{\Phi \in \mathscr{S} \mid \phi_{i}=1\right\} .
$$

It is maximal for $\Phi=\left(\frac{1}{3}, \frac{1}{3}, \frac{1}{3}\right)$. In sub-domains where only two phases are present it coincides with a standard doublewell potential. Both $W$ and $\partial_{\phi_{i}} W$ are Lipschitz in $\mathscr{S}$, since for $\Phi_{1}, \Phi_{2} \in \mathscr{S}$, where $\Phi_{j}=\left(\phi_{j, 1}, \phi_{j, 2}, \phi_{j, 3}\right)$ for $j=1,2$, it holds

$$
\begin{aligned}
& \left|W\left(\Phi_{1}\right)-W\left(\Phi_{2}\right)\right| \leqslant 18 \sum_{i \in I}\left|\phi_{1, i}-\phi_{2, i}\right|, \\
& \left|\partial_{\phi_{i}}\left(W\left(\Phi_{1}\right)-W\left(\Phi_{2}\right)\right)\right| \leqslant 42\left|\phi_{1, i}-\phi_{2, i}\right| .
\end{aligned}
$$

Figure 2.4a depicts a contour plot of $W$ in $\mathscr{S}$. Both, $U$ resp. $W$ are particular cases of the free energy resp. potential developed in Boyer \& Lapuerta (2006); Boyer et al. (2010). The idea in these papers is to include in the evolution equation for the phase-field $\phi_{i}$ not only the derivative $\partial_{\phi_{i}} W$, but also the other derivatives, $\partial_{\phi_{j}} W$ for $j \in I \backslash\{i\}$. In this way, $\phi_{1}+\phi_{2}+\phi_{3}=1$ holds almost everywhere. In Boyer \& Lapuerta (2006); Boyer et al. (2010), a Cahn-Hilliard flow model is developed. The drawback in this approach is that it does not guarantee that $\phi_{i} \in[0,1]$ for $i \in I$. As will be shown below, having $\phi_{1} \in[0,1]$ is essential for the well-posedness of the evolution equation for the concentration. Therefore, Allen-Cahn type equations are preferred here.

The idea of phase-field modeling is to replace sharp interfaces by diffuse interface regions. Specifically, for any $i, j \in I$ with $i<j$, a diffuse interface $\Gamma_{i j}^{d}(t)$ approximating the sharp-interface $\Gamma_{i j}^{s}(t)$ is characterized by

$$
\Gamma_{i j}^{d}(t):=\left\{x \in \mathscr{P} \mid \Phi(t, x) \in \bar{\Gamma}_{i j}^{d}\right\}, \text { for } t \in \overline{J(T)},
$$

with the diffuse interface states

$$
\bar{\Gamma}_{i j}^{d}:=\left\{\Phi \in \mathscr{S} \mid \phi_{i}+\phi_{j}=1, \phi_{i}, \phi_{j} \in(0,1)\right\} .
$$

Recalling (2.12), for $i \in I$ we define the pure state $P_{i}^{d}(t)$ as

$$
P_{i}^{d}(t):=\left\{x \in \mathscr{P} \mid \Phi(t, x) \in \bar{P}_{i}^{d}\right\}, \text { for } t \in \overline{J(T)} .
$$

In this context, within the diffuse interface $\Gamma_{12}^{d}(t)$ separating the pure sub-domains $P_{1}^{d}(t)$ and $P_{2}^{d}(t)$, the phase-field $\phi_{1}$ varies smoothly from 1 (taken in $P_{1}^{d}(t)$ ) to 0 (taken in $P_{2}^{d}(t)$ ). Similarly, since $\phi_{2}$ approximates the characteristic function of $P_{2}^{d}(t)$, it varies smoothly from 0 in $P_{1}^{d}(t)$ to 1 in $P_{2}^{d}(t)$.

The force $f_{1}$, which models the precipitation process of the solute in $P_{1}^{d}(t)$ at the boundary of $P_{3}^{d}(t)$, must vanish in the sub-domains where $\phi_{1}$ or $\phi_{3}$ vanish: in the diffuse interfaces $\Gamma_{12}^{d}(t)$ and $\Gamma_{23}^{d}(t)$, and in the pure regions $P_{1}^{d}(t), P_{2}^{d}(t)$ and $P_{3}^{d}(t)$. A suitable choice is

$$
f_{1}: \mathscr{S} \times \mathbb{R} \rightarrow \mathbb{R}, f_{1}(\Phi, u)=\alpha \xi \gamma_{13}(\Phi) f(u),
$$


where $f(u)$ is the precipitation rate-function defined in (2.5). The function $\gamma_{13}$,

$$
\gamma_{13}: \mathscr{S} \rightarrow[0,1], \gamma_{13}(\Phi)=4 \phi_{1} \phi_{3},
$$

vanishes in

$$
\bar{\Gamma}_{12}^{d} \cup \bar{\Gamma}_{23}^{d} \cup \bar{P}_{1}^{d} \cup \bar{P}_{2}^{d} \cup \bar{P}_{3}^{d} .
$$

Therefore, the indicator $\gamma_{13} \geqslant 0$ vanishes everywhere in the pore-space except for the regions where both $\phi_{1}$ and $\phi_{3}$ are non-zero. It is maximal for $\Phi=\left(\frac{1}{2}, 0, \frac{1}{2}\right)$, that is exactly in the middle of $\Gamma_{13}^{d}(t)$. Figure $2.4 \mathrm{~b}$ depicts a contour plot of $\gamma_{13}(\Phi)$ in $\mathscr{S}$. The parameters $\alpha$ and $\xi$ are parameters of the evolution equation to be given in the following.

Since the diffuse interfaces $\Gamma_{12}^{d}(t)$ and $\Gamma_{23}^{d}(t)$ are not driven by precipitation, and since the effect of the precipitation in the interface region $\Gamma_{13}^{d}(t)$ on $\phi_{1}$ must be contrary to the effect on $\phi_{3}$, the driving forces $f_{2}$ and $f_{3}$ are defined by

$$
f_{2}(\Phi, u)=0, f_{3}(\Phi, u)=-f_{1}(\Phi, u)=-\alpha \xi \gamma_{13}(\Phi) f(u) .
$$

Furthermore, define

$$
G_{i}(\Phi, u)=\left(\sum_{j \in I \backslash\{i\}}\left(\partial_{\phi_{i}}-\partial_{\phi_{j}}\right) W(\Phi)\right)+f_{i}(\Phi, u) \text { for } i \in I .
$$

The forces $f_{i}$ are locally Lipschitz. Especially, if $\Phi_{1}, \Phi_{2} \in \mathscr{S}$ and $\left|u_{1}\right|,\left|u_{2}\right| \leqslant d<\infty$ then

$$
\begin{aligned}
& \left|f_{1}\left(\Phi_{1}, u_{1}\right)-f_{1}\left(\Phi_{2}, u_{2}\right)\right| \\
\leqslant & 4 \alpha \xi\left(\left|u_{1}-u_{2}\right|+c_{u, 0}\left(d+c_{u, 1}\right) \sum_{j=1,3}\left|\phi_{1, j}-\phi_{2, j}\right|\right) .
\end{aligned}
$$

Hence, the equations (2.13) and (2.22) yield Lipschitz estimates for the functions $G_{i}, i \in I$, in $\mathscr{S} \times[-d, d]$.

Summarizing the discussion above, this section is concluded with the following problem for the phase-fields

Problem 2.2 Given the parameters $\alpha, \xi>0$, the concentration $u: \overline{J(T)} \times \mathscr{P} \rightarrow \mathbb{R}$, the functions $G_{i}(\Phi, u)$ defined by (2.21), the potential $W(\Phi)$ defined by (2.10), the driving forces $f_{i}(\Phi, u)$ defined by (2.17) and (2.20), and the initial condition $\Phi_{0}=\left(\phi_{1,0}, \phi_{2,0}, \phi_{3,0}\right): \mathscr{P} \rightarrow \mathscr{S}$, find the phase-fields $\Phi=\left(\phi_{1}, \phi_{2}, \phi_{3}\right)$ satisfying

$$
\begin{array}{ll}
\alpha \xi^{2} \partial_{t} \phi_{i}-\frac{3}{4} \xi^{2} \Delta \phi_{i}+G_{i}(\Phi, u)=0 & \text { in } J(T) \times \mathscr{P}, \\
-\frac{3}{4} \xi^{2} \nabla \phi_{i} \cdot n_{\mathscr{P}}=0 & \text { on } J(T) \times \Gamma_{\mathscr{P}}, \\
\Phi(0, x)=\Phi_{0}(x) & \text { in } \mathscr{P} .
\end{array}
$$

The solute concentration $u$ is assumed sufficiently regular here. The parameter $\alpha>0$ is a time relaxation depending on the constant surface tension $\sigma>0$. In Section 2.3, the choice $\frac{3}{4 \sigma}$ is made explicitly. The width of the diffuse interface regions is proportional to the second parameter, $\xi>0$. This is explained by a competition between the Laplacian in (2.23) and the sum of the potential derivatives (see (2.21)).

In Section 2.2.4 we prove that a weak solution $\Phi$ to Problem 2.2 satisfies $\Phi \in \mathscr{S}$ almost everywhere in $J(T) \times \mathscr{P}$ if $\Phi_{0} \in \mathscr{S}$ for a.e. $x \in \mathscr{P}$. Furthermore, in Section 2.3 it is shown that, passing $\xi \rightarrow 0$, the limit-interfaces $\Gamma_{i j}^{\ell}(t)$ of $\Gamma_{i j}^{d}(t)$ are evolving with normal velocities satisfying the kinetic conditions (2.3) of the sharp-interface model.

\subsubsection{The evolution equation for the solute concentration $u$}

Having introduced the phase-fields, we now draw the attention to the solute concentration. In the sharp-interface approach, its evolution is described by (2.2), which is valid in the a-priori unknown time-space region $J(T) \times P_{1}^{s}(t)$. The aim is to approximate this by an equation that is valid in the whole pore-space $J(T) \times \mathscr{P}$. The transformation is motivated by the Diffuse Domain Approximation (DDA) method introduced in Kockelkoren et al. (2003); Li et al. (2009). Additional source terms approximate the Rankine-Hugoniot conditions at the sharp interfaces $\Gamma_{12}^{s}(t)$ and $\Gamma_{13}^{s}(t)$, that is at the interfaces separating the regions occupied by $P_{1}^{s}(t)$ and $P_{2}^{s}(t)$ as well as by $P_{1}^{s}(t)$ and $P_{3}^{s}(t)$. Similar to Section 2.2.1, here the phase-fields are assumed to be given and admissible, $\Phi \in \mathscr{S}$ in $\overline{J(T)} \times \mathscr{P}$.

REMARK 2.3 Our approach is motivated by the DDA model in Li et al. (2009), which we shortly review in this remark. For $M_{1}(t) \subset M \subset \mathbb{R}^{d}$, and $t \in[0, T]$, let $\phi:[0, T] \times M \rightarrow[0,1]$ be a smooth approximation to the characteristic function 
$\chi(t)$ of $M_{1}(t)$, and let $n_{M}$ resp. $n_{M_{1}}$ be the outward-normal to $\partial M$ resp. $\partial M_{1}$. The phase-field $\phi$ is either given or described by an evolution equation. The DDA model reads: find $u_{d}:[0, T] \times M \rightarrow \mathbb{R}$ satisfying

$$
\begin{array}{lr}
\partial_{t}\left((\phi+\delta) u_{d}\right)-\nabla \cdot\left((\phi+\delta) \nabla u_{d}\right)-g_{d}|\nabla \phi|=0 & \text { in }(0, T] \times M \\
-(\phi+\delta) \nabla u_{d} \cdot n_{M}=0 & \text { on }(0, T] \times \partial M
\end{array}
$$

Employing a matched asymptotic expansion, and letting the width $\xi>0$ of the interface region approach 0 , in $\mathrm{Li}$ et al. (2009) it is shown that $u_{d}$ converges to the solution $u_{s}:[0, T] \times M_{1}([0, T]) \rightarrow \mathbb{R}$ of the sharp-interface model

$$
\begin{array}{lc}
\partial_{t} u_{s}-\Delta u_{s}=0 & \text { in }(0, T] \times M_{1}(t), \\
-\nabla u_{s} \cdot n_{M_{1}}=v^{n} u_{s}-g_{s} & \text { on }(0, T] \times \partial M_{1}(t) .
\end{array}
$$

Here $v^{n}$ stands for the outward normal velocity of the evolving boundary $\partial M_{1}(t)$. The function $g_{s}:(0, T] \times \partial M_{1}(t) \rightarrow \mathbb{R}$ is defined only on $\partial M_{1}(t)$, whereas $g_{d}:(0, T] \times M \rightarrow \mathbb{R}$ appearing in the DDA model is an extension of $g_{s}$ to the whole space. Finally, the regularization parameter $\delta>0$ appearing in the DDA-model ensures the well-posedness, as $\phi$ might vanish somewhere in $M$. The matched asymptotic expansion in Li et al. (2009) uses the choice $\delta=\xi$. Note that $u_{d}$ is defined in the whole spatial domain $M$, while $u_{s}$ is only defined in the subdomain $M_{1} \subset M$.

We adopt this idea in the present framework and assume $\Phi: \overline{J(T)} \times \mathscr{P} \rightarrow \mathscr{S}$ given and having sufficient regularity; a phase-field $\phi_{i}: J(T) \times \mathscr{P} \rightarrow[0,1]$ approximates the characteristic function $\chi_{i}(t)$ of the region $P_{i}^{s}(t)(i \in I)$. To approximate the Rankine-Hugoniot conditions in $\left(2.2_{3,4}\right)$ at the sharp-interfaces $\Gamma_{12}^{s}(t)$ and $\Gamma_{13}^{s}(t)$, a function $\bar{g}: J(T) \times$ $\mathscr{P} \rightarrow \mathbb{R}$ is defined to interpolating between the phases. This interpolation is needed in regions of triple junctions, where the three sharp-interfaces $\Gamma_{12}^{s}(t), \Gamma_{13}^{s}(t)$ and $\Gamma_{23}^{s}(t)$ meet (see Figures 2.1 on page 4 and 2.2 on page 5). In the diffuse setting, the sharp interfaces $\Gamma_{i j}^{s}(t)$ are approximated by the the diffuse interfaces $\Gamma_{i j}^{d}(t)$ characterized in (2.14), (2.15). In the diffuse triple junctions $\Gamma_{123}^{d}(t)$, approximating $\Gamma_{123}^{s}(t)$, all three phase-fields $\phi_{1}, \phi_{2}$ and $\phi_{3}$ exist. Hence,

$$
\Gamma_{123}^{d}(t)=\left\{x \in \mathscr{P} \mid \Phi(t, x) \in \bar{\Gamma}_{123}^{d}\right\}, \text { for } t \in \overline{J(T)},
$$

where

$$
\bar{\Gamma}_{123}^{d}=\left\{\Phi \in \mathscr{S} \mid \phi_{1}, \phi_{2}, \phi_{3} \in(0,1)\right\} .
$$

An interpolation is required only at locations of these diffuse triple junctions.

For $\delta \geqslant 0$ define

$$
g_{\delta}: \mathscr{S} \rightarrow[0,1], g_{\delta}(\Phi)= \begin{cases}1 & \text { if } \delta=0 \text { and } \Phi=(1,0,0), \\ \frac{\phi_{3}}{\phi_{2}+\phi_{3}+\delta}=\frac{\phi_{3}}{1-\phi_{1}+\delta} & \text { else. }\end{cases}
$$

Recall that $\Phi \in \mathscr{S}$ yields $\phi_{1}+\phi_{2}+\phi_{3}=1$ and $\phi_{i} \geqslant 0$ for $i \in I$. Therefore, $g_{\delta}$ is defined everywhere in $\mathscr{S}$. As depicted in Figure 2.4c, $g_{d}$ interpolates linearly in $\bar{\Gamma}_{123}^{d}$ between (approx.) 1 in $\bar{\Gamma}_{13}^{d}$ and 0 in $\bar{\Gamma}_{12}^{d}$.

The diffuse phase interface $\Gamma_{1}^{d}(t)$, where for $i \in I$

$$
\bar{\Gamma}_{i}^{d}:=\left\{\Phi \in \mathscr{S} \mid \phi_{i} \in(0,1)\right\} \text { and } \Gamma_{i}^{d}(t):=\left\{x \in \mathscr{P} \mid \Phi(t, x) \in \bar{\Gamma}_{i}^{d}\right\} \text {, for } t \in \overline{J(T)},
$$

of the pure phase $P_{1}^{d}(t)$ moves with the normal velocity $v_{1}^{n}=\frac{\partial_{t} \phi_{1}}{\left|\nabla \phi_{1}\right|}$ into the direction of the outer-normal $n_{1}=-\frac{\nabla \phi_{1}}{\left|\nabla \phi_{1}\right|}$. Therefore, the function $\bar{g}$, finally defined by

$$
\bar{g}: J(T) \times \mathscr{P} \rightarrow \mathbb{R}, \bar{g}(t, x)=\rho v_{1}^{n} g_{\delta}(\Phi(t, x)),
$$

interpolates between the two Rankine-Hugoniot interface conditions of the sharp-interface model (2.2). Multiplying $\bar{g}$ and $\left|\nabla \phi_{1}\right|$ results in

$$
\bar{g}\left|\nabla \phi_{1}\right|=\rho v_{1}^{n} g_{\delta}(\Phi)\left|\nabla \phi_{1}\right|=\rho \frac{\partial_{t} \phi_{1}}{\left|\nabla \phi_{1}\right|} g_{\delta}(\Phi)\left|\nabla \phi_{1}\right|=\rho g_{\delta}(\Phi) \partial_{t} \phi_{1}
$$

In sum, for given phase-fields $\Phi \in \mathscr{S}$, these considerations yield the following DDA model for the concentration $u$ of the dissolved precipitate in the fluid $P_{1}$. 
Problem 2.3 Given the constant diffusion coefficient $D>0$, the smooth phase-fields $\Phi: \overline{J(T)} \times \mathscr{P} \rightarrow \mathscr{S}$, the regularization parameter $\delta>0$, the concentration $\rho>0$ of particles in the precipitate $P_{3}$, the interpolation-function $g_{\delta}(\Phi)$ defined by (2.28), and the initial condition $u_{0}: \mathscr{P} \rightarrow \mathbb{R}$, find the concentration $u$ satisfying

$$
\begin{array}{ll}
\partial_{t}\left(\left(\phi_{1}+\delta\right) u\right)-D \nabla \cdot\left(\left(\phi_{1}+\delta\right) \nabla u\right)-\rho g_{\delta}(\Phi) \partial_{t} \phi_{1}=0 & \text { in } J(T) \times \mathscr{P}, \\
-D\left(\phi_{1}+\delta\right) \nabla u \cdot n_{\mathscr{P}}=0 & \text { on } J(T) \times \Gamma_{\mathscr{P}} \\
u(0, x)=u_{0}(x) & \text { in } \mathscr{P} .
\end{array}
$$

It is important to note that though the unknown concentration $u$ is determined in the whole domain, it describes only the concentration of particles in the region $P_{1}^{d}(t)$ occupied by the fluid $P_{1}$. Contrarily, the function $\tilde{u}(\Phi, u, \rho)=$ $\phi_{1} u+\phi_{3} \rho$ describes the concentration in the whole pore-space, that is $\tilde{u}=u$ in $P_{1}^{d}(t), \tilde{u}=0$ in $P_{2}^{d}(t)$ and $\tilde{u}=\rho$ in $P_{3}^{d}(t)$.

REMARK 2.4 In case no fluid $P_{2}$ exists, meaning that $\phi_{2} \equiv 0$ in $\overline{J(T)} \times \mathscr{P}$, we can replace $g_{\delta}(\Phi)$ by 1 . Then the evolution equation in Problem 2.3 becomes

$$
\partial_{t}\left(\left(\phi_{1}+\delta\right)(u-\rho)\right)-D \nabla \cdot\left(\left(\phi_{1}+\delta\right) \nabla u\right)=0
$$

This equation has the same structure as a variant of the diffusion equation for the concentration of particles in the phase-field model introduced in Noorden \& Eck (2011),

$$
0=\partial_{t}((k(\phi)+\delta)(u-\rho))-D \nabla \cdot((k(\phi)+\delta) \nabla u) .
$$

The above is obtained from the model in Noorden \& Eck (2011) if the unknown $u=\frac{c-\rho}{k(\phi)+\delta}+\rho$ replaces the original unknown concentration $c$. Also note that the phase-field $\phi$ varies smoothly from -1 for the liquid to 1 for the precipitate, and that $k(\phi)$ varies smoothly from 1 for the liquid to 0 for the precipitate. Consequently, $k(\phi)$ is the counterpart of the present function $\phi_{1}$ - both indicating the fluid phase containing solute. As mentioned, the distinguishing feature of the present model when compared to the one in Noorden \& Eck (2011) is that it deals with three phases instead of only two. Therefore, the present model can be seen as an extension of the two-phase model in Noorden \& Eck (2011) to a multi-phase setting.

\subsubsection{The combined pore-scale phase-field model}

The Problems 2.2 and 2.3 assume that either the phase-fields $\Phi$ or the concentration $u$ are given. Their coupling results in the following problem.

Problem 2.4 Given the parameters $\alpha, \xi, D, \delta, \rho>0$, the functions $G_{i}(\Phi, u)$ defined by $(2.21)$, the potential $W(\Phi)$ defined by (2.10), the driving forces $f_{i}(\Phi, u)$ defined by (2.17) and (2.20), the interpolation-function $g_{\delta}(\Phi)$ defined by (2.28), and the initial conditions $\Phi_{0}=\left(\phi_{1,0}, \phi_{2,0}, \phi_{3,0}\right): \mathscr{P} \rightarrow \mathscr{S}$ and $u_{0}: \mathscr{P} \rightarrow \mathbb{R}$, find the phase-fields $\Phi=\left(\phi_{1}, \phi_{2}, \phi_{3}\right)$ and the concentration $u$ satisfying

$$
\begin{array}{lr}
\alpha \xi^{2} \partial_{t} \phi_{i}-\frac{3}{4} \xi^{2} \Delta \phi_{i}+G_{i}(\Phi, u)=0 & \text { in } J(T) \times \mathscr{P}, \\
\partial_{t}\left(\left(\phi_{1}+\delta\right) u\right)-D \nabla \cdot\left(\left(\phi_{1}+\delta\right) \nabla u\right)-\rho g_{\delta}(\Phi) \partial_{t} \phi_{1}= & \text { in } J(T) \times \mathscr{P}, \\
-\frac{3}{4} \xi^{2} \nabla \phi_{i} \cdot n_{\mathscr{P}}=0 & \text { on } J(T) \times \Gamma_{\mathscr{P}} \\
-D\left(\phi_{1}+\delta\right) \nabla u \cdot n_{\mathscr{P}}=0 & \text { on } J(T) \times \Gamma_{\mathscr{P}} \\
\Phi(0, x)=\Phi_{0}(x) & \text { in } \mathscr{P}, \\
u(0, x)=u_{0}(x) & \text { in } \mathscr{P} .
\end{array}
$$

\subsubsection{Analysis of the Problem 2.2}

In this subsection it is proven that, firstly, the subspace $\mathscr{S}$ of the admissible states of $\Phi$ is an invariant subspace of the solution operator $\Phi_{0} \mapsto \Phi$ of Problem 2.2, secondly, if a classical solution $\Phi$ to Problem 2.2 exists and the given data $u$ is bounded, then the increase of the system's free energy $U$ (defined in (2.9)) is bounded as well.

Let $\langle\cdot, \cdot\rangle_{\mathscr{P}}$ denote the dual pairing in $H^{1}(\mathscr{P})$. Using standard notation, define the function-spaces

$$
\begin{aligned}
& \mathscr{V}(T):=L_{2}\left(J(T) ; H^{1}(\mathscr{P})\right) \cap H^{1}\left(J(T) ; H^{1}(\mathscr{P})^{\star}\right), \\
& \mathscr{W}(T):=\left\{V=\left(v_{1}, v_{2}, v_{3}\right) \in\left(L_{\infty}(J(T) \times \mathscr{P})\right)^{3} \mid V(t, x) \in \mathscr{S} \text { for a.e. }(t, x) \in J(T) \times \mathscr{P}\right\},
\end{aligned}
$$


and the abbreviations

$$
\begin{aligned}
\langle v, w\rangle & =\langle v, w\rangle_{J(T) \times \mathscr{P}}=\int_{J(T)}\langle v, w\rangle_{\mathscr{P}} d t, \\
(v, w) & =(v, w)_{J(T) \times \mathscr{P}}=\int_{J(T) \times \mathscr{P}} v w d x d t, \\
\|\cdot\|_{r, T} & =\|\cdot\|_{L_{r}(J(T) \times \mathscr{P})} \text { for } 1 \leqslant r \leqslant \infty .
\end{aligned}
$$

The Problem 2.2 has the following weak formulation.

Problem 2.5 Given the parameters $\alpha, \xi>0$, the concentration $u \in L_{\infty}(J(T) \times \mathscr{P})$, the functions $G_{i}(\Phi, u)$ defined by (2.21), the potential $W(\Phi)$ defined by (2.10), the driving forces $f_{i}(\Phi, u)$ defined by (2.17) and (2.20), and the initial condition $\Phi_{0}=\left(\phi_{1,0}, \phi_{2,0}, \phi_{3,0}\right) \in \mathscr{S}$ for a.e. $x \in \mathscr{P}$, find the phase-fields $\Phi=\left(\phi_{1}, \phi_{2}, \phi_{3}\right) \in \mathscr{V}(T)^{3}$ such that the initial condition is satisfied and such that

$$
\left\langle\alpha \xi^{2} \partial_{t} \phi_{i}, v_{i}\right\rangle+\left(\frac{3}{4} \xi^{2} \nabla \phi_{i}, \nabla v_{i}\right)+\left(G_{i}(\Phi, u), v_{i}\right)=0
$$

holds true for any test-functions $v_{i} \in L_{2}\left(J(T) ; H^{1}(\mathscr{P})\right)$ and for all $i \in I$.

Theorem 2.6 The Problem 2.5 is uniquely solvable. The unique weak solution $\Phi$ satisfies

$$
\Phi \in \mathscr{V}(T)^{3} \cap C\left(\overline{J(T)} ; L_{2}(\mathscr{P})\right)^{3} \cap \mathscr{W}(T)
$$

Proof. This proof follows the outline of the proof of Theorem 2 in Section 9.2 of Evans (2010). It relies on Banach's Fixed Point Theorem applied to the solution operator $\mathscr{T}$, defined with the help of a linearized version of Problem 2.5 and on the space

$$
X_{T}:=C\left(\overline{J(T)} ; L_{2}(\mathscr{P})\right)^{3} \cap \mathscr{W}(T)
$$

with the norm

$$
\|V\|_{X_{T}}:=\sum_{i \in I} \max _{t \in \overline{J(T)}}\left\|v_{i}(t)\right\|_{L_{2}(\mathscr{P})}, V=\left(v_{1}, v_{2}, v_{3}\right) \in X_{T}
$$

Step one. Linearization: instead of the non-linear system (2.38), consider its linearized version below. Given $u \in L_{\infty}(J(T) \times \mathscr{P})$ and the initial data in Problem 2.5, for prescribed input data $\hat{\Phi}=\left(\hat{\phi}_{1}, \hat{\phi}_{2}, \hat{\phi}_{3}\right) \in \mathscr{W}(T)$ and a sufficiently large constant $L>0$ that will be specified later, find $\Phi=\left(\phi_{1}, \phi_{2}, \phi_{3}\right) \in \mathscr{V}(T)^{3}$ such that

$$
\left\langle\alpha \xi^{2} \partial_{t} \phi_{i}, v_{i}\right\rangle+\left(\frac{3}{4} \xi^{2} \nabla \phi_{i}, \nabla v_{i}\right)+\left(L \phi_{i}, v_{i}\right)=\left(L \hat{\phi}_{i}, v_{i}\right)-\left(G_{i}(\hat{\Phi}, u), v_{i}\right)
$$

holds true for any test-functions $v_{i} \in L_{2}\left(J(T) ; H^{1}(\mathscr{P})\right)$ and for all $i \in I$. Since $G_{i}(\hat{\Phi}, u) \in L_{2}(J(T) \times \mathscr{P})$, Theorem 10.3 and Lemma 10.4 of Renardy \& Rogers (2004) (or Lemma 7.3 of Roubicek (2005)) provide the existence and uniqueness of the weak solution triple $\Phi=\left(\phi_{1}, \phi_{2}, \phi_{3}\right) \in\left(\mathscr{V}(T) \cap C\left(\overline{J(T)} ; L_{2}(\mathscr{P})\right)\right)^{3}$ of $(2.42)$.

Step two. Regularity: the solution $\Phi$ lies in $\mathscr{W}(T)$. We let $\chi_{t_{0}}$ be the indicator-function of the time-interval $J\left(t_{0}\right)=\left(0, t_{0}\right] \subseteq(0, T]=J(T)$ and $v=\left(\phi_{1}+\phi_{2}+\phi_{3}-1\right)$. Testing in (2.42) with $v_{i}=v \chi_{t_{0}}$ and adding the result for $i=1,2,3$, since $v(0)=0, \sum_{i \in I}\left(\hat{\phi}_{i}, v\right)=(1, v)$ and $\sum_{i \in I}\left(G_{i}(\hat{\Phi}, u), v\right)=0$, yields

$$
\begin{aligned}
0 & =\sum_{i \in I}\left\langle\alpha \xi^{2} \partial_{t} \phi_{i}, v\right\rangle+\sum_{i \in I}\left(\frac{3}{4} \xi^{2} \nabla \phi_{i}, \nabla v\right)+\sum_{i \in I}\left(L \phi_{i}, v\right)-\sum_{i \in I}\left(L \hat{\phi}_{i}, v\right)+\sum_{i \in I}\left(G_{i}(\hat{\Phi}, u), v\right) \\
& =\frac{\alpha \xi^{2}}{2}\left(\left\|v\left(t_{0}\right)\right\|_{L_{2}(\mathscr{P})}^{2}-\|v(0)\|_{L_{2}(\mathscr{P})}^{2}\right)+\frac{3}{4} \xi^{2}\|\nabla v\|_{2, t_{0}}^{2}+L \sum_{i \in I}\left(\phi_{i}, v\right)-L(1, v) \\
& =\frac{\alpha \xi^{2}}{2}\left\|v\left(t_{0}\right)\right\|_{L_{2}(\mathscr{P})}^{2}+\frac{3}{4} \xi^{2}\|\nabla v\|_{2, t_{0}}^{2}+L\|v\|_{2, t_{0}}^{2} .
\end{aligned}
$$

Hence, $\sum_{i \in I} \phi_{i}(t, x)=1$ for a.e. $(t, x) \in J(T) \times \mathscr{P}$. 
12 of 31

Observe that, since $\hat{\Phi} \in \mathscr{S}$,

$$
\begin{aligned}
\sum_{j \in I \backslash\{i\}}\left(\partial_{\phi_{i}}-\partial_{\phi_{j}}\right) W(\hat{\Phi})= & 8\left(\hat{\phi}_{i}-3 \hat{\phi}_{i}^{2}+2 \hat{\phi}_{i}^{3}\right)-4\left(\hat{\phi}_{j}-3 \hat{\phi}_{j}^{2}+2 \hat{\phi}_{j}^{3}\right) \\
& -4\left(\left(1-\hat{\phi}_{i}-\hat{\phi}_{j}\right)-3\left(1-\hat{\phi}_{i}-\hat{\phi}_{j}\right)^{2}+2\left(1-\hat{\phi}_{i}-\hat{\phi}_{j}\right)^{3}\right) \\
= & 8\left(\hat{\phi}_{i}-3 \hat{\phi}_{i}^{2}+2 \hat{\phi}_{i}^{3}\right)-4\left(\hat{\phi}_{j}-3 \hat{\phi}_{j}^{2}+2 \hat{\phi}_{j}^{3}\right) \\
& -4\left(-\hat{\phi}_{i}+3 \hat{\phi}_{i}^{2}-2 \hat{\phi}_{i}^{3}-\hat{\phi}_{j}+3 \hat{\phi}_{j}^{2}-2 \hat{\phi}_{j}^{3}+6 \hat{\phi}_{i} \hat{\phi}_{j}-6 \hat{\phi}_{i}^{2} \hat{\phi}_{j}-6 \hat{\phi}_{i} \hat{\phi}_{j}^{2}\right) \\
= & 12\left(\hat{\phi}_{i}-3 \hat{\phi}_{i}^{2}+2 \hat{\phi}_{i}^{3}-2 \hat{\phi}_{i} \hat{\phi}_{j}+2 \hat{\phi}_{i}^{2} \hat{\phi}_{j}+2 \hat{\phi}_{i} \hat{\phi}_{j}^{2}\right) \\
= & 12 \hat{\phi}_{i}(\underbrace{1-3 \hat{\phi}_{i}+2 \hat{\phi}_{i}^{2}}_{\leqslant 1}+2 \hat{\phi}_{j}(\underbrace{-1+\hat{\phi}_{i}+\hat{\phi}_{j}}_{\leqslant 0})) \\
\leqslant & 12 \hat{\phi}_{i} .
\end{aligned}
$$

Defining the cut-off function $[\cdot]_{-}: x \mapsto[x]_{-}=\min (0, x)$, testing in (2.42) with $v_{i}=\left[\phi_{i}\right]_{-} \chi_{t_{0}}$, since $\phi_{i}(0) \geqslant 0$ a straightforward calculations gives

$$
\frac{\alpha \xi^{2}}{2}\left\|\left[\phi_{i}\left(t_{0}\right)\right]_{-}\right\|_{L_{2}(\mathscr{P})}^{2}+\frac{3}{4} \xi^{2}\left\|\nabla\left[\phi_{i}\right]_{-}\right\|_{2, t_{0}}^{2}+L\left\|\left[\phi_{i}\right]_{-}\right\|_{2, t_{0}}^{2}=\left(L \hat{\phi}_{i}-G_{i}(\hat{\Phi}, u),\left[\phi_{i}\right]_{-} \chi_{t_{0}}\right)
$$

We now take

$$
L \geqslant 12+4 \alpha \xi c_{u, 0}\left(\|u\|_{\infty, T}+c_{u, 1}\right)
$$

Using the definition of $G_{i}$ and the inequality (2.44) yields immediately

$$
\begin{aligned}
& \frac{\alpha \xi^{2}}{2}\left\|\left[\phi_{i}\left(t_{0}\right)\right]_{-}\right\|_{L_{2}(\mathscr{P})}^{2}+\frac{3}{4} \xi^{2}\left\|\nabla\left[\phi_{i}\right]_{-}\right\|_{2, t_{0}}^{2}+L\left\|\left[\phi_{i}\right]_{-}\right\|_{2, t_{0}}^{2} \\
\leqslant & \left(\hat{\phi}_{i}(-L+12+4 \alpha \xi|f(u)|),\left|\left[\phi_{i}\right]_{-}\right| \chi_{t_{0}}\right) \\
\leqslant & \left(\hat{\phi}_{i}\left(-L+12+4 \alpha \xi c_{u, 0}\left(\|u\|_{\infty, T}+c_{u, 1}\right)\right),\left|\left[\phi_{i}\right]_{-}\right| \chi_{t_{0}}\right) .
\end{aligned}
$$

Due to (2.46), the right hand side is negative, implying that $\phi_{i}(t, x) \geqslant 0$ for a.e. $(t, x) \in J(T) \times \mathscr{P}$. In summary, $\Phi=\left(\phi_{1}, \phi_{2}, \phi_{3}\right) \in \mathscr{W}(T)$.

Step three. The estimates: given $\hat{\Phi} \in X_{T}$ and with $\Phi=\left(\phi_{1}, \phi_{2}, \phi_{3}\right)$ solving the linear system (2.42), the solution operator

$$
\mathscr{T}: X_{T} \rightarrow X_{T}, \mathscr{T}(\hat{\Phi})=\Phi
$$

is well defined, and $\mathscr{T}(\hat{\Phi}) \in \mathscr{W}(T)$. Further we prove that $\mathscr{T}$ is a strict contraction if $T>0$ is small enough.

In this sense we consider two admissible input data $\hat{\Phi}^{k}=\left(\hat{\phi}_{k, 1}, \hat{\phi}_{k, 2}, \hat{\phi}_{k, 3}\right)(k=1,2)$ and let $\Phi^{k}=\mathscr{T}\left(\hat{\Phi}_{k}\right)$ be the corresponding solutions for the same initial condition $\Phi_{0}$ and concentration $u$. With $i \in I$ we define

$$
\bar{\phi}_{i}:=\phi_{1, i}-\phi_{2, i}, \quad \text { and } \quad \bar{\phi}_{i}:=\bar{\phi}_{1, i}-\bar{\phi}_{2, i},
$$

and take $v_{i}=\bar{\phi}_{i} \chi_{t_{0}}$ as test functions in (2.42) for both input data $\hat{\Phi}^{k}$. Subtract the resulting and and then summing up these differences for $i \in I$ yields

$$
\begin{aligned}
& \sum_{i \in I}\left(\alpha \xi^{2}\left\langle\partial_{t} \bar{\phi}_{i}, \bar{\phi}_{i} \chi_{t_{0}}\right\rangle+\frac{3}{4} \xi^{2}\left(\nabla \bar{\phi}_{i}, \nabla \bar{\phi}_{i} \chi_{t_{0}}\right)+L\left(\bar{\phi}_{i}, \bar{\phi}_{i} \chi_{t_{0}}\right)\right) \\
= & \sum_{i \in I} L\left(\overline{\hat{\phi}}_{i}, \bar{\phi}_{i} \chi_{t_{0}}\right)-\sum_{i=1,3}\left(f_{i}\left(\hat{\Phi}_{1}, u\right)-f_{i}\left(\hat{\Phi}_{2}, u\right), \bar{\phi}_{i} \chi_{t_{0}}\right) \\
& -\sum_{i \in I} \sum_{j \in I \backslash\{i\}}\left(\left(\partial_{\phi_{i}}-\partial_{\phi_{j}}\right)\left(W\left(\hat{\Phi}_{1}\right)-W\left(\hat{\Phi}_{2}\right)\right), \bar{\phi}_{i} \chi_{t_{0}}\right) .
\end{aligned}
$$

Since the initial conditions and the input data $u$ are the same for both solution triples, the Lipschitz continuity of $\partial_{\phi_{i}} W(\Phi)$ and $f_{i}(\Phi, u)$ (see (2.13) and (2.22)), by the inequalities of Hölder and Young the following holds for an 
arbitrary $\eta>0$

$$
\begin{aligned}
& \sum_{i \in I}\left(\alpha \xi^{2}\left\|\bar{\phi}_{i}\left(t_{0}\right)\right\|_{L_{2}(\mathscr{P})}^{2}+\frac{3}{4} \xi^{2}\left\|\nabla \bar{\phi}_{i}\right\|_{2, t_{0}}^{2}+L\left\|\bar{\phi}_{i}\right\|_{2, t_{0}}^{2}\right) \\
\leqslant & L \sum_{i \in I}\left\|\overline{\hat{\phi}}_{i}\right\|_{2, t_{0}}\left\|\bar{\phi}_{i}\right\|_{2, t_{0}}+4 \alpha \xi c_{u, 0}\left(\|u\|_{\infty, t_{0}}+c_{u, 1}\right) \sum_{i, j=1,3}\left\|\overline{\hat{\phi}}_{j}\right\|_{2, t_{0}}\left\|\bar{\phi}_{i}\right\|_{2, t_{0}} \\
& +42 \sum_{i \in I} \sum_{j \in I \backslash\{i\}}\left(\left\|\overline{\hat{\phi}}_{i}\right\|_{2, t_{0}}+\left\|\overline{\hat{\phi}}_{j}\right\|_{2, t_{0}}\right)\left\|\bar{\phi}_{i}\right\|_{2, t_{0}} \\
\leqslant & C\left(L, 4 \alpha \xi c_{u, 0}\left(\|u\|_{\infty, t_{0}}+c_{u, 1}\right), 42, \eta\right) \sum_{i \in I}\left\|\overline{\hat{\phi}}_{i}\right\|_{2, t_{0}}^{2}+\eta \sum_{i \in I}\left\|\bar{\phi}_{i}\right\|_{2, t_{0}}^{2} .
\end{aligned}
$$

Taking $\eta \leqslant L$ results in

$$
\sum_{i \in I}\left\|\bar{\phi}_{i}\left(t_{0}\right)\right\|_{L_{2}(\mathscr{P})}^{2} \leqslant C \sum_{i \in I}\left\|\overline{\hat{\phi}}_{i}\right\|_{2, t_{0}}^{2} \leqslant C T \sum_{i \in I} \max _{t \in \overline{J(T)}}\left\|\overline{\hat{\phi}}_{i}(t)\right\|_{L_{2}(\mathscr{P})}^{2}
$$

for each $t_{0} \in \overline{J(T)}$. The constant $C>0$ is independent of the initial condition $\Phi_{0}$, the solutions $\Phi_{1}$ and $\Phi_{2}$ and the input data $\hat{\Phi}_{1}$ and $\hat{\Phi}_{2}$. Maximizing the left-hand side with respect to $t_{0}$ yields

$$
\left\|\mathscr{T}\left(\hat{\Phi}_{1}\right)-\mathscr{T}\left(\hat{\Phi}_{2}\right)\right\|_{X_{T}}=\left\|\Phi_{1}-\Phi_{2}\right\|_{X_{T}} \leqslant \sqrt{C T}\left\|\hat{\Phi}_{1}-\hat{\Phi}_{2}\right\|_{X_{T}} .
$$

Consequently, the solution operator $\mathscr{T}$ is a strict contraction as long as $T>0$ is so small such that $\sqrt{C T}<1$.

Step four. The contraction: fix $t_{0} \in J(T)$ such that $\sqrt{C t_{0}}<1$ and hence $\mathscr{T}: X_{t_{0}} \rightarrow X_{t_{0}}$ is a strict contraction. Banach Fixed Point Theorem provides the existence of a solution to the non-linear weak Problem 2.5 in the time-space cylinder $J\left(t_{0}\right) \times \mathscr{P}$. Since the choice of $t_{0}$ is independent of the solution and the initial data, this argument can be repeated with the solution at $t_{0}$ as initial data, providing the existence of a solution on the interval $\left[t_{0}, 2 t_{0}\right]$ and so on until reaching the time $T$. Therefore, a solution to the weak Problem 2.5 exists in the space $\mathscr{W}(T) \cap \mathscr{V}(T)^{3} \cap C\left(\overline{J(T)} ; L_{2}(\mathscr{P})\right)^{3}$. Finally, uniqueness follows by Gronwall's inequality, applied to (2.51).

Theorem 2.6 also shows that the weak solution $\Phi$ of Problem 2.5 is in an admissible state almost everywhere in the time-space cylinder. The following theorem shows that, if the solution $\Phi$ is a classical solution (i.e. solving Problem 2.2), then the free energy $U$ defined in (2.9) has a bounded growth rate.

THEOREM 2.7 Assume that Problem 2.2 has a classical solution $\Phi=\left(\phi_{1}, \phi_{2}, \phi_{3}\right)$, then the system has a free energy $U$ with bounded growth. Specifically, there exist the constants $C_{1}, C_{2}>0$ s.t.

$$
\frac{d}{d t} U(\Phi) \leqslant C_{1}\left\|\phi_{1} \phi_{3}\left(u-c_{u, 1}\right)\right\|_{L_{2}(\mathscr{P})}^{2}-C_{2} \sum_{i \in I}\left\|\partial_{t} \phi_{i}\right\|_{L_{2}(\mathscr{P})}^{2} .
$$

Moreover, if $f(u)=0\left(c_{u, 0}=0\right.$ or $\left.u=c_{u, 1}\right)$ in regions of $\mathscr{P}$ where $\phi_{1} \phi_{3}>0$, then the free energy is decreasing.

The last statement in Theorem 2.7 can be interpreted in the following sense: the free energy is decreasing if the solute concentration takes the equilibrium value everywhere in regions where both phases fields $\phi_{1}$ and $\phi_{3}$ coexist. In other words, the free energy is decreasing if no dissolution and precipitation is encountered in the diffuse interface region between water and precipitate.

Proof. Using the evolution equation in Problem 2.2, since $\partial_{t} \phi_{i}=-\sum_{j \in I \backslash\{i\}} \partial_{t} \phi_{j}$ for $i \in I$, the definition of the driving forces (2.17) and (2.20), and Young's inequality for an arbitrary $\eta>0$ yield

$$
\begin{aligned}
\frac{d}{d t} U(\Phi) & =\int_{\mathscr{P}} 3 \partial_{t} W(\Phi)+\frac{3}{8} \xi^{2} \sum_{i \in I} \partial_{t}\left|\nabla \phi_{i}\right|^{2} d x \\
& =\int_{\mathscr{P}} \sum_{i \in I}\left(3 \partial_{\phi_{i}} W(\Phi) \partial_{t} \phi_{i}+\frac{3}{4} \xi^{2} \nabla \phi_{i} \cdot \nabla\left(\partial_{t} \phi_{i}\right)\right) d x \\
& =\int_{\mathscr{P}} \sum_{i \in I}\left(\partial_{\phi_{i}} W(\Phi) \sum_{j \in I \backslash\{i\}}\left(\partial_{t} \phi_{i}-\partial_{t} \phi_{j}\right)+\frac{3}{4} \xi^{2} \nabla \phi_{i} \cdot \nabla\left(\partial_{t} \phi_{i}\right)\right) d x \\
& =\int_{\mathscr{P}} \sum_{i \in I}\left(\sum_{j \in I \backslash\{i\}}\left(\partial_{\phi_{i}}-\partial_{\phi_{j}}\right) W(\Phi)-\frac{3}{4} \xi^{2} \Delta \phi_{i}\right) \partial_{t} \phi_{i} d x \\
& =\int_{\mathscr{P}} \sum_{i \in I}\left(-f_{i}(\Phi, u) \partial_{t} \phi_{i}-\alpha \xi^{2}\left(\partial_{t} \phi_{i}\right)^{2} d x\right) d x \\
& =\int_{\mathscr{P}} 4 \alpha \xi \phi_{1} \phi_{3} f(u) \partial_{t}\left(\phi_{3}-\phi_{1}\right)+\sum_{i \in I}-\alpha \xi^{2}\left(\partial_{t} \phi_{i}\right)^{2} d x \\
& \leqslant \frac{1}{4 \eta}\left\|4 \phi_{1} \phi_{3} f(u)\right\|_{L_{2}(\mathscr{P})}^{2}-\left(\alpha-\eta \alpha^{2}\right) \xi^{2} \sum_{i \in I}\left\|\partial_{t} \phi_{i}\right\|_{L_{2}(\mathscr{P})}^{2} \\
& \leqslant \frac{c_{u, 0}}{4 \eta}\left\|u-c_{u, 1}\right\|_{L_{2}(\mathscr{P})}^{2}-\left(\alpha-\eta \alpha^{2}\right) \xi^{2} \sum_{i \in I}\left\|\partial_{t} \phi_{i}\right\|_{L_{2}(\mathscr{P})}^{2} .
\end{aligned}
$$


Consequently, with $0<\eta \leqslant \alpha^{-1}$, the boundedness of $u$ provides an upper bound for the increase of the free energy. In particular, if $f(u)=0$, that is if $c_{u, 0}=0$ or $u=c_{u, 1}$, in regions of $\mathscr{P}$ where $\phi_{1} \phi_{3}>0$ then the free energy decreases monotonically.

REMARK 2.5 For given phase fields, the existence and uniqueness of a weak solution $u$ to Problem 2.3 for the concentration $u$ is a standard result. The analysis of the coupled system in Problem 2.4 instead will be part of future research.

\subsection{The asymptotic behavior for $\xi \rightarrow 0$}

In this subsection the formal asymptotic limit of Problem 2.4 for the parameter $\xi \rightarrow 0$ is analyzed. Since $\xi$ is proportional to the width of the interface regions in Problem 2.4, the matched asymptotic expansion method describes the transition from the phase-field model to its sharp interface limit. As usual in the literature, e.g. Bronsard \& Reitich (1993), the method realizes a spatial decomposition: firstly, the outer regions far away from the interfaces, secondly, the inner regions near the interfaces of two phases far away from the other interfaces and, thirdly, the inner regions near triple junctions where three interfaces meet. The variables are expanded in powers of $\xi$ in each region. In a region where two expansions are valid, the expansions are matched.

Let $\left(\Phi_{\xi}, u_{\xi}\right)=\left(\phi_{1, \xi}, \phi_{2, \xi}, \phi_{3, \xi}, u_{\xi}\right)$ be a sufficiently smooth solution to Problem 2.4 with the parameter $\xi>0$ and with $\delta=\xi$. The following considerations show that if $\xi \rightarrow 0$ then the pore-space is subdivided into regions where $\Phi_{\xi} \approx \bar{P}_{1}^{d}=(1,0,0), \Phi_{\xi} \approx \bar{P}_{2}^{d}=(0,1,0)$ or $\Phi_{\xi} \approx \bar{P}_{3}^{d}=(0,0,1)$, that is into regions occupied by an approximately pure fluid $P_{1}$, by an approximately pure fluid $P_{2}$ and into regions occupied by an approximately pure precipitate $P_{3}$. In the limit, the interfaces $\Gamma_{12}^{d}(t)$ and $\Gamma_{23}^{d}(t)$ evolve with speed equal to their mean curvature. The evolution of interface $\Gamma_{13}^{d}(t)$ is influenced by the precipitation reaction. It evolves with the normal velocity $-\sigma \kappa_{13}-f(c)$. Hence, these limit-interfaces evolve with normal velocities that fulfill the kinetic conditions (2.3) in the sharp-interface setting. Due to the fact that the potential $W(\Phi)$ is symmetric, and consequently the surface tensions of the three interfaces are equal, Bronsard \& Reitich (1993) shows that the angles between the limit-interfaces in triple junctions must be of $120^{\circ}$ in a two-dimensional spatial setting. Furthermore, it is shown that the limit-solution $u_{\xi}$ satisfies in the region occupied by the approximately pure fluid $P_{1}$ the evolution equation, and on the limit-interfaces the Rankine-Hugoniot conditions of the sharp-interface problem 2.1.

For ease of presentation, throughout this subsection the interpolation function $g_{\delta}(\Phi)$ of Problem 2.4 is replaced by $g_{0}(\Phi)$, both defined in (2.28). It is $g_{0}(\Phi)=1$ in $\Gamma_{13}^{d}(t)$ and $g_{0}(\Phi)=0$ in $\Gamma_{12}^{d}(t)$.

\subsubsection{The expansions and their matching conditions}

The outer (that is, away from interfaces) expansions $\left(\Phi^{\text {out }}, u^{\text {out }}\right)$ to the solution $\left(\Phi_{\xi}, u_{\xi}\right)$ are given by

$$
\begin{aligned}
& \Phi^{\text {out }}(t, x)=\left(\phi_{1}^{\text {out }}(t, x), \phi_{2}^{\text {out }}(t, x), \phi_{3}^{\text {out }}(t, x)\right)=\left(\sum_{i=0}^{\infty} \xi^{i} \phi_{1, i}^{\text {out }}(t, x), \sum_{i=0}^{\infty} \xi^{i} \phi_{2, i}^{\text {out }}(t, x), \sum_{i=0}^{\infty} \xi^{i} \phi_{3, i}^{\text {out }}(t, x)\right), \\
& u^{\text {out }}(t, x)=\sum_{i=0}^{\infty} \xi^{i} u_{i}^{\text {out }}(t, x) .
\end{aligned}
$$

Near an interface far away from the other interfaces, the coordinates $x \in \mathscr{P}$ are transformed into local curvilinear coordinates. The transformation is derived from the level sets $\Gamma_{i, \xi}^{d}(t), i \in I$,

$$
\Gamma_{i, \xi}^{d}(t)=\left\{x \in \mathscr{P} \mid \phi_{i, \xi}(x)=0.5\right\}
$$

It is assumed that the level sets $\Gamma_{i, \xi}^{d}(t)$ are sufficiently smooth and for $\xi \rightarrow 0$ smoothly approach limit-interfaces $\Gamma_{i}^{\ell}(t)$, where the index $\ell$ denotes 'limit' as $s$ denotes 'sharp' and $d$ 'diffuse'. Exemplarily considered is the situation for the interface $\Gamma_{13}^{d}(t)$ that separates the two fluids $P_{1}^{d}(t)$ and $P_{3}^{d}(t)$ and that is far away from the other interfaces. It is assumed that $\Gamma_{13, \xi}^{d}(t)=\Gamma_{1, \xi}^{d}(t)=\Gamma_{3, \xi}^{d}(t) \rightarrow \Gamma_{13}^{\ell}(t)$ smoothly for $\xi \rightarrow 0$. For $x \in \mathscr{P}$, let $r(t, x)$ denote the signed distance from $x$ to $\Gamma_{13, \xi}^{d}(t)$, where $r$ is negative for $x$ in $P_{1}$ and positive in $P_{3}$. In addition, let $s$ be a measure of arc length from some fixed point, and let $y_{\xi}(t, s)$ be the point on $\Gamma_{13, \xi}^{d}(t)$ at arc length $s$. Then $(r, s)$ is a local orthogonal coordinate system and any point $x$ in a neighborhood of $\Gamma_{13, \xi}^{d}(t)$ is representable by

$$
x=y_{\xi}(t, s)+r n_{\xi}(t, s),
$$


where $n_{\xi}(t, s)$ is the normal to $\Gamma_{13, \xi}^{d}(t)$ in $y_{\xi}(t, s)$ pointing into $P_{3}^{d}(t)$. In Caginalp \& Fife (1988) it is shown that the coordinates $(r, s)=(r, s)(t, x)$ satisfy

$$
|\nabla r|=1, \nabla r \cdot \nabla s=0, \partial_{t} r=-v^{n}, \Delta r=\frac{\kappa+2 \Pi r}{1+\kappa r+\Pi r^{2}},
$$

where $v^{n}, \kappa$ and $\Pi$ denote the normal velocity, the mean and the Gaussian curvature of the moving interface $\Gamma_{13, \xi}^{d}(t)$. It is assumed that the normal velocity $v^{n}$ and the mean curvature $\kappa$ satisfy the expansions

$$
v^{n}=v_{0}^{n}+\mathscr{O}(\xi), \kappa=\kappa_{0}+\mathscr{O}(\xi)
$$

where $v_{0}^{n}$ and $\kappa_{0}$ denote the normal velocity and the mean curvature of $\Gamma_{13}^{\ell}(t)$. Finally introduce the coordinates $z=\xi^{-1} r$ and the inner expansions $\Phi^{i n}$ of $\Phi_{\xi}$ and $u^{i n}$ of $u_{\xi}$,

$$
\begin{aligned}
& \Phi^{i n}(t, x)=\left(\phi_{1}^{i n}(t, x), \phi_{2}^{i n}(t, x), \phi_{3}^{i n}(t, x)\right)=\left(\sum_{i=0}^{\infty} \xi^{i} \phi_{1, i}^{i n}(t, x), \sum_{i=0}^{\infty} \xi^{i} \phi_{2, i}^{i n}(t, x), \sum_{i=0}^{\infty} \xi^{i} \phi_{3, i}^{i n}(t, x)\right), \\
& u^{i n}(t, z, s)=\sum_{i=0}^{\infty} \xi^{i} u_{i}^{i n}(t, z, s) .
\end{aligned}
$$

In an overlapping region the inner and the outer expansions shall describe the same function. This is realized by matching conditions. For that purpose, the outer expansions are written in terms of the local coordinates $(t, z, s)$, where the outer normal $n_{\xi}(t, s)$ and the point $y_{\xi}(t, s)$ (see (2.58)) are expanded as follows

$$
\begin{aligned}
& n_{\xi}(t, s)=\sum_{i=0}^{\infty} \xi^{i} n_{i}(t, s), \\
& y_{\xi}(t, s)=\sum_{i=0}^{\infty} \xi^{i} n_{i-1}(t, s) y_{i}(t, s), n_{-1} \equiv 1 .
\end{aligned}
$$

Let $y_{-1 / 2}$ denote the limit at the free interface $\Gamma_{13, \xi}^{d}(t)$ from the side of $P_{1}(r, z<0)$ and $y_{+1 / 2}$ the one on the right $(r, z>0)$, that is on the side of $P_{3}$. In Caginalp \& Fife (1988) it is shown that equating the inner- and the outerexpansions yields the following matching conditions

$$
\begin{aligned}
& \lim _{z \rightarrow \pm \infty} \phi_{1,0}^{\text {in }}(t, z, s)=\phi_{1,0}^{\text {out }}\left(t, y_{ \pm 1 / 2}\right) \\
& \lim _{z \rightarrow \pm \infty}\left(\phi_{1,1}^{\text {in }}(t, z, s)-\left(\nabla \phi_{1,0}^{\text {out }}\left(t, y_{ \pm 1 / 2}\right) \cdot n_{0}\right)\left(y_{1}+z\right)\right)=\phi_{1,1}^{\text {out }}\left(t, y_{ \pm 1 / 2}\right), \\
& \lim _{z \rightarrow \pm \infty} \partial_{z} \phi_{1,0}^{\text {in }}(t, z, s)=0 \\
& \lim _{z \rightarrow \pm \infty} \partial_{z} \phi_{1,1}^{\text {in }}(t, z, s)=\nabla \phi_{1,0}^{\text {out }}\left(t, y_{ \pm 1 / 2}\right) \cdot n_{0},
\end{aligned}
$$

and

$$
\begin{aligned}
& \lim _{z \rightarrow \pm \infty} u_{0}^{\text {in }}(t, z, s)=u_{0}^{\text {out }}\left(t, y_{ \pm 1 / 2}\right) \\
& \lim _{z \rightarrow \pm \infty}\left(u_{1}^{\text {in }}(t, z, s)-\left(\nabla u_{0}^{\text {out }}\left(t, y_{ \pm 1 / 2}\right) \cdot n_{0}\right)\left(y_{1}+z\right)\right)=u_{1}^{\text {out }}\left(t, y_{ \pm 1 / 2}\right) \\
& \lim _{z \rightarrow \pm \infty} \partial_{z} u_{0}^{\text {in }}(t, z, s)=0 \\
& \lim _{z \rightarrow \pm \infty} \partial_{z} u_{1}^{\text {in }}(t, z, s)=\nabla u_{0}^{\text {out }}\left(t, y_{ \pm 1 / 2}\right) \cdot n_{0}
\end{aligned}
$$

For the inner expansions $w^{i n}(t, z, s), w^{i n} \in\left\{\phi_{1}^{i n}, \phi_{2}^{i n}, \phi_{3}^{i n}, u^{i n}\right\}$, the following relations hold

$$
\begin{aligned}
& \partial_{t} w^{i n}=-\frac{1}{\xi} v^{n} \partial_{z} w^{i n}+\left(\partial_{t}+\partial_{t} s \cdot \nabla_{s}\right) w^{i n}, \\
& \nabla_{x} \cdot\left(K \nabla_{x} w^{i n}\right)=\frac{1}{\xi^{2}} \partial_{z} \cdot\left(K \partial_{z} w^{i n}\right)+\frac{1}{\xi}\left(\Delta_{x} r\right) K \partial_{z} w^{i n}+\mathscr{O}(1),
\end{aligned}
$$

where $\Delta_{x} r=\kappa_{0}+\mathscr{O}\left(\xi^{1}\right)$ holds in a small neighborhood of $\Gamma_{13, \xi}^{d}(t)$, and where $K$ is a sufficiently smooth diffusion tensor.

\subsubsection{The outer expansions}

Substituting the outer expansions (2.56) into the first evolution equation of (2.35) of Problem 2.4 yields a sequence of problems. For $i \in I$ the $\mathscr{O}(1)$ problem consists of the equation

$$
\sum_{j \in I \backslash\{i\}}\left(\partial_{\phi_{i}}-\partial_{\phi_{j}}\right) W\left(\phi_{1,0}^{\text {out }}, \phi_{2,0}^{\text {out }}, \phi_{3,0}^{\text {out }}\right)=0 \text { in } J(T) \times \mathscr{P} \text {. }
$$


16 of 31

The solutions that are considered are

$$
\Phi_{0}^{\text {out }}=\left(\phi_{1,0}^{\text {out }}, \phi_{2,0}^{\text {out }}, \phi_{3,0}^{\text {out }}\right) \in\left\{\bar{P}_{1}^{d}, \bar{P}_{2}^{d}, \bar{P}_{3}^{d}\right\}=\{(1,0,0),(0,1,0),(0,0,1)\} .
$$

The zeros $\bar{P}_{i}^{d}, i \in I$, minimize the potential in (2.66). The remaining zeros $\tilde{\Phi}$, as e.g. $\left(\frac{1}{2}, \frac{1}{2}, 0\right)$ and $\left(\frac{1}{3}, \frac{1}{3}, \frac{1}{3}\right)$, are not considered because they are unstable due to

$$
\partial_{\phi_{i}} \sum_{j \in I \backslash\{i\}}\left(\partial_{\phi_{i}}-\partial_{\phi_{j}}\right) W(\tilde{\Phi})<0 \text { for } i \in I .
$$

Therefore, substituting the outer expansions into the first evolution equation of (2.35) subdivides the pore-space $\mathscr{P}$ into $P_{1^{-}}, P_{2^{-}}$and $P_{3}$-regions.

The problem at $\mathscr{O}\left(\xi^{1}\right)$ for $i \in I$ reads

$$
\begin{aligned}
& 8 \phi_{i, 1}^{\text {out }}\left(1-6 \phi_{i, 0}^{\text {out }}+6\left(\phi_{i, 0}^{\text {out }}\right)^{2}\right)-4 \sum_{\ell \in I \backslash\{i\}} \phi_{\ell, 1}^{\text {out }}\left(1-6 \phi_{\ell, 0}^{\text {out }}+6\left(\phi_{\ell, 0}^{\text {out }}\right)^{2}\right) \\
= & \begin{cases}-4 \alpha \phi_{1,0}^{\text {out }} \phi_{3,0}^{\text {out }} f\left(u_{0}^{\text {out }}\right), & \text { if } i=1, \\
0, & \text { if } i=2, \\
4 \alpha \phi_{1,0}^{\text {out }} \phi_{3,0}^{\text {out }} f\left(u_{0}^{\text {out }}\right), & \text { if } i=3 .\end{cases}
\end{aligned}
$$

If $\Phi_{0}^{\text {out }} \in\left\{\bar{P}_{1}^{d}, \bar{P}_{2}^{d}, \bar{P}_{3}^{d}\right\}$, the left-hand side is equal to $8 \phi_{i, 1}^{\text {out }}-4 \sum_{\ell \in I \backslash\{i\}} \phi_{\ell, 1}^{\text {out }}$ and the right-hand side vanishes. Consequently, if $\Phi_{0}^{\text {out }} \in\left\{\bar{P}_{1}^{d}, \bar{P}_{2}^{d}, \bar{P}_{3}^{d}\right\}$ then the three equations yield $\phi_{1,1}^{\text {out }}=\phi_{2,1}^{\text {out }}=\phi_{3,1}^{\text {out }}$. The condition $\sum_{j=0}^{\infty} \xi^{j} \sum_{i \in I} \phi_{i, j}^{\text {out }}=1$ yields at $\mathscr{O}\left(\xi^{1}\right)$ the condition $\sum_{i \in I} \phi_{i, 1}^{\text {out }}=0$. In summary, if $\Phi_{0}^{\text {out }} \in\left\{\bar{P}_{1}^{d}, \bar{P}_{2}^{d}, \bar{P}_{3}^{d}\right\}$, these considerations yield $\phi_{1,1}^{\text {out }}=$ $\phi_{2,1}^{\text {out }}=\phi_{3,1}^{\text {out }}=0$.

Define

$$
P_{i}^{\text {out }}(t):=\left\{x \in \mathscr{P} \mid \Phi_{0}^{\text {out }}(t, x) \in \bar{P}_{i}^{d}\right\}, \text { for } t \in \overline{J(T)} .
$$

As already shown in Li et al. (2009), if $\Phi_{0}^{\text {out }}=\bar{P}_{1}^{d}$, that is if $\phi_{1,0}^{\text {out }}=1$, substituting the outer expansions into the second evolution equation of (2.35) yields, since $\delta=\xi$, at $\mathscr{O}\left(\xi^{0}\right)$ the equation

$$
\partial_{t} u_{0}^{\text {out }}-D \Delta u_{0}^{\text {out }}=0 \text { in } J(T) \times P_{1}^{\text {out }}(t),
$$

due to $\partial_{t} \phi_{1,0}^{\text {out }}=0$ and $g_{0}(1,0,0)=1$ (recall that $g_{\delta}(\Phi)$ is replaced by $g_{0}(\Phi)$ for ease of presentation). Hence, the sharp-interface model's evolution equation in (2.2) for the concentration in $J(T) \times P_{1}^{s}(t)$ is recovered. reads

If $\Phi_{0}^{\text {out }} \in\left\{\bar{P}_{2}^{\text {out }}, \bar{P}_{3}^{\text {out }}\right\}$, no equation at $\mathscr{O}\left(\xi^{0}\right)$ results from the substitution, since $\phi_{1,0}^{\text {out }}=0$. At $\mathscr{O}\left(\xi^{1}\right)$ the problem

$$
\partial_{t} u_{0}^{\text {out }}-D \Delta u_{0}^{\text {out }}=0 \text { in } J(T) \times P_{2}^{\text {out }}(t) \text { and in } J(T) \times P_{3}^{\text {out }}(t),
$$

since $\delta=\xi$ and due to the fact that $\phi_{1,0}^{\text {out }}, \phi_{1,1}^{\text {out }}=0$ if $\Phi_{0}^{\text {out }} \in\left\{\bar{P}_{2}^{d}, \bar{P}_{3}^{d}\right\}$.

\subsubsection{The inner expansions $\phi_{i}^{i n}$ at $\mathscr{O}\left(\xi^{0}\right)$ near $\Gamma_{12, \xi}^{d}(t), \Gamma_{13, \xi}^{d}(t)$ and $\Gamma_{23, \xi}^{d}(t)$}

Near the interface of $\Gamma_{13, \xi}^{d}(t)$ that separates $P_{1}^{\text {out }}(t)$ and $P_{3}^{\text {out }}(t)$ in a region far away from the remaining interface regions $\Gamma_{12, \xi}^{d}(t), \Gamma_{23, \xi}^{d}(t)$ and $\Gamma_{123, \xi}^{d}(t)$, it is assumed that $\phi_{2}^{i n}=0$. Consequently, substituting the inner expansions (2.61) into the first evolution equation of (2.35) results for $i=1$ in an equation that is, due to $\phi_{2}^{i n}=0$ and hence $\phi_{3}^{i n}=1-\phi_{1}^{i n}$, equivalent to

$$
\alpha \xi^{2} \partial_{t} \phi_{1}^{i n}-\frac{3}{4} \xi^{2} \Delta \phi_{1}^{i n}+6 p^{\prime}\left(\phi_{1}^{i n}\right)+4 \xi \alpha \sqrt{p\left(\phi_{1}^{i n}\right)} f(u)=0
$$

where $p(\phi)=\phi^{2}(1-\phi)^{2}$. Following the argumentation in Noorden \& Eck (2011), that is substituting the inner expansions (2.61) into (2.73) and collecting the terms of order $\xi^{0}$ yields the second-order ordinary differential equation

$$
-\frac{3}{4} \partial_{z}^{2} \phi_{1,0}^{i n}+6 p^{\prime}\left(\phi_{1,0}^{i n}\right)=0 .
$$


Due to the first matching condition in (2.63), equation (2.74) is supplemented by the interface conditions

$$
\phi_{1,0}^{i n}(t,-\infty, s)=1, \phi_{1,0}^{i n}(t, \infty, s)=0 .
$$

Due to the condition $\phi_{1}^{i n}(t, 0, s)=\frac{1}{2}$ and the assumption that $\Gamma_{13, \xi}^{d}(t)$ converges for $\xi \rightarrow 0$ to $\Gamma_{13}^{\ell}(t), \phi_{1,0}^{i n}(t, 0, s)=\frac{1}{2}$ as well. Since $p\left(\frac{1}{2}+z\right)=p\left(\frac{1}{2}-z\right)$ and since $p$ is non-negative, $\frac{3}{8} \partial_{z}\left(\partial_{z} \phi_{1,0}^{i n}\right)^{2}=6 \partial_{z} p\left(\phi_{1,0}^{i n}\right)$, and due to the first and the third matching conditions in (2.63), the second-order problem (2.74) reduces to the initial value problem

$$
\partial_{z} \phi_{1,0}^{i n}=-4 \sqrt{p\left(\phi_{1,0}^{i n}\right)}=-4 \phi_{1,0}^{i n}\left(1-\phi_{1,0}^{i n}\right), \phi_{1,0}^{i n}(0)=\frac{1}{2} .
$$

Separation of variables yields the unique, strictly decreasing solution

$$
\phi_{1,0}^{i n}(t, z, s)=\phi_{1,0}^{i n}(z)=\frac{1}{1+\exp (4 z)} .
$$

As a consequence,

$$
\phi_{3,0}^{i n}(t, z, s)=1-\phi_{1,0}^{i n}(t, z, s)=\frac{1}{1+\exp (-4 z)} .
$$

Similar calculations can be carried out near the interfaces $\Gamma_{12, \xi}^{d}(t)$ and $\Gamma_{23, \xi}^{d}(t)$. They result in equivalent expressions for $\phi_{i, 0}^{i n}(t, z, s)$ and $\phi_{j, 0}^{i n}(t, z, s)$ near $\Gamma_{i j, \xi}^{d}(t)$.

\subsubsection{The inner expansions $u^{\text {in }}$ at $\mathscr{O}\left(\xi^{-2}\right)$ near $\Gamma_{12, \xi}^{d}(t)$ and $\Gamma_{13, \xi}^{d}(t)$}

The following argumentation holds in the neighborhood of $\Gamma_{12, \xi}^{d}(t)$ as well as in the one of $\Gamma_{13, \xi}^{d}(t)$. For $i \in\{2,3\}$ fixed, substituting the inner expansions (2.61) in the neighborhood of $\Gamma_{1 i, \xi}^{d}(t)$ into the second evolution equation of (2.35) results at $\mathscr{O}\left(\xi^{-2}\right)$ in the equation

$$
\partial_{z}\left(\phi_{1,0}^{i n} \partial_{z} u_{0}^{i n}\right)=0 \text {. }
$$

As already shown in Li et al. (2009), integration from $-\infty$ to $z \in \mathbb{R}$, the condition $\phi_{1,0}^{i n}(t, z, s)>0$ for all $z \in \mathbb{R}$, and the third matching condition in (2.64) yield

$$
\partial_{z} u_{0}^{i n}=0
$$

\subsubsection{The inner expansions $\phi_{i}^{i n}$ at $\mathscr{O}\left(\xi^{1}\right)$ near $\Gamma_{12, \xi}^{d}(t), \Gamma_{13, \xi}^{d}(t)$ and $\Gamma_{23, \xi}^{d}(t)$}

Following the argumentation in Noorden \& Eck (2011), that is substituting the inner expansions (2.61) near the interface $\Gamma_{13, \xi}^{d}(t)$ into (2.73) and collecting the terms of order $\xi^{1}$ yields the equation

$$
\underbrace{\left(-\frac{3}{4} \partial_{z}^{2}+6 p^{\prime \prime}\left(\phi_{1,0}^{i n}\right)\right)}_{=: L\left(\phi_{1,0}^{i n}\right)=L} \phi_{1,1}^{i n}=\underbrace{\left(\alpha v_{0}^{n}+\frac{3}{4} \kappa_{0}\right) \partial_{z} \phi_{1,0}^{i n}-4 \alpha \phi_{1,0}^{i n}\left(1-\phi_{1,0}^{i n}\right) f\left(u_{0}^{i n}\right)}_{=: A\left(\phi_{1,0}^{i n}\right)} .
$$

The equation is supplemented by the condition that $\phi_{1}( \pm \infty)$ is bounded because of the second matching condition (2.63). The operator $L$ is a Fredholm operator of index zero. Therefore, the Fredholm alternative yields that the equation has a solution iff $A\left(\phi_{1,0}^{i n}\right)$ is orthogonal to the finite-dimensional kernel of $L$. Because $\partial_{z} \phi_{1,0}^{i n} \in \operatorname{ker}(L)$ (see (2.74)), because $\partial_{z} \phi_{1,0}^{i n}=-4 \phi_{1,0}^{i n}\left(1-\phi_{1,0}^{i n}\right)$ holds true, and because $v_{0}^{n}, \kappa_{0}$ and $u_{0}^{i n}$ are independent of $z$, the solvability condition implies

$$
\begin{aligned}
0 & =\int_{-\infty}^{\infty} A\left(\phi_{1,0}^{i n}\right) \partial_{z} \phi_{1,0}^{i n} d z \\
& =\left(\alpha v_{0}^{n}+\frac{3}{4} \kappa_{0}\right) \int_{-\infty}^{\infty}\left(\partial_{z} \phi_{1,0}^{i n}\right)^{2} d z-4 \alpha f\left(u_{0}^{i n}\right) \int_{-\infty}^{\infty} \phi_{1,0}^{i n}\left(1-\phi_{1,0}^{i n}\right) \partial_{z} \phi_{1,0}^{i n} d z \\
& =-4\left(\alpha v_{0}^{n}+\frac{3}{4} \kappa_{0}+\alpha f\left(u_{0}^{i n}\right)\right) \int_{-\infty}^{\infty} \phi_{1,0}^{i n}\left(1-\phi_{1,0}^{i n}\right) \partial_{z} \phi_{1,0}^{i n} d z \\
& =-4\left(\alpha v_{0}^{n}+\frac{3}{4} \kappa_{0}+\alpha f\left(u_{0}^{i n}\right)\right) \int_{1}^{0} x(1-x) d x \\
& =\frac{2}{3}\left(\alpha v_{0}^{n}+\frac{3}{4} \kappa_{0}+\alpha f\left(u_{0}^{i n}\right)\right)
\end{aligned}
$$


Set

$$
\alpha:=\frac{3}{4} \sigma^{-1},
$$

where $\sigma>0$ describes the surface tension in the sharp-interface problem 2.1 with the kinetic conditions (2.3) of the moving interfaces. The linear growth of the rate-function $f$ and the first matching condition in (2.64) yield

$$
v_{0}^{n}=-\sigma \kappa_{0}-f\left(u_{0}^{\text {out }}\left(t, y_{-1 / 2}\right)\right) \text { for } z \rightarrow-\infty .
$$

Near the interfaces $\Gamma_{12, \xi}^{d}(t)$ and $\Gamma_{23, \xi}^{d}(t)$ a similar argumentation leads to the equation

$$
\left(-\frac{3}{4} \partial_{z}^{2}+6 p^{\prime \prime}\left(\phi_{2,0}^{i n}\right)\right) \phi_{2,1}^{i n}=\left(\alpha v_{0}^{n}+\frac{3}{4} \kappa_{0}\right) \partial_{z} \phi_{2,0}^{i n}
$$

for $\phi_{2,1}^{i n}$. Consequently,

$$
v_{0}^{n}=-\sigma \kappa_{0} .
$$

Therefore, the moving interfaces' kinetic conditions (2.3) of the sharp-interface model are recovered at $\mathscr{O}\left(\xi^{1}\right)$.

\subsubsection{The inner expansions $u^{\text {in }}$ at $\mathscr{O}\left(\xi^{-1}\right)$ near $\Gamma_{12, \xi}^{d}(t)$ and $\Gamma_{13, \xi}^{d}(t)$}

Substituting the inner expansions (2.61) in the neighborhood of $\Gamma_{13, \xi}^{d}(t)$ into the second evolution equation of (2.35) results at $\mathscr{O}\left(\xi^{-1}\right)$ in the equation

$$
-D \partial_{z} \cdot\left(\phi_{1,0}^{i n} \partial_{z} u_{1}^{i n}\right)=v_{0}^{n}\left(u_{0}^{i n}-\rho\right) \partial_{z} \phi_{1,0}^{i n},
$$

since $\partial_{z} u_{0}^{i n}=0$, and since $g_{0}\left(\Phi_{0}^{i n}\right)=1$ due to $\phi_{2,0}^{i n}=0$ (recall that $g_{\delta}(\Phi)$ is replaced by $g_{0}(\Phi)$ for ease of presentation). As already shown in Li et al. (2009), since $v_{0}^{n}, u_{0}^{i n}$ and $\rho$ are independent of $z$, integrating from $-\infty$ to $\infty$, and the first matching condition in (2.63) imply

$$
-D \partial_{z} u_{1}^{i n}=v_{0}^{n}\left(u_{0}^{i n}-\rho\right) .
$$

The first and the fourth matching condition in (2.64) yield

$$
-D \nabla u_{0}^{\text {out }}\left(t, y_{-1 / 2}\right) \cdot n_{0}=v_{0}^{n}\left(u_{0}^{\text {out }}\left(t, y_{-1 / 2}\right)-\rho\right) .
$$

Since $g_{0}\left(\Phi_{0}^{i n}\right)=0$ in the neighborhood of $\Gamma_{12, \xi}^{d}(t)$, a similar argumentation as in the neighborhood of $\Gamma_{13, \xi}^{d}(t)$ yields in the neighborhood of $\Gamma_{12, \xi}^{d}(t)$ the equation

$$
-D \nabla u_{0}^{\text {out }}\left(t, y_{-1 / 2}\right) \cdot n_{0}=v_{0}^{n} u_{0}^{\text {out }}\left(t, y_{-1 / 2}\right) .
$$

Thus, the sharp-interface model's Rankine-Hugoniot conditions in (2.2) at $\Gamma_{12}^{s}(t)$ and $\Gamma_{13}^{s}(t)$ are recovered at $\mathscr{O}\left(\xi^{-1}\right)$.

\subsubsection{Conclusion}

The asymptotic analysis showed that the solution to the limit problem 2.4 for $\xi \rightarrow 0$ approximates the solution of the sharp-interface problem 2.1 with the kinetic conditions (2.3) for the moving interfaces. The analysis of the asymptotic behavior of the solution $u_{\xi}$ near the interface $\Gamma_{23, \xi}^{d}(t)$ as well as near triple junctions is a part of future research.

\section{Upscaling}

Theoretically, the phase-field Problem 2.4 discussed in the previous section approximates the evolution of the three phases, and the diffusion process of the concentration in an arbitrarily domain with an arbitrary number of phase transition regions. In this respect, the model does not change if only a small number of phase transitions are encountered, or if this number is very large. The latter situation is encountered e.g. in a porous medium. If the processes are viewed 
at the pore scale, then Problem 2.4 is a pore scale model defined in a very complex domain, with pores surrounding an extremely large number of solid particles, the grains. Phase transitions can take place within each pore, which makes computations at this scale prohibitive: the discretization should be fine enough to resolve accurately these phase transitions, which leads to an enormous number of unknowns. In this case, homogenization offers the right balance between accuracy and computability: the resulting models are describing the averaged behaviour of the system accurately, and provide sufficient information to recover the details at the pore scale. For details on homogenization in porous media we refer to Hornung (1997).

Below we apply homogenization techniques to derive the upscaled (Darcy scale) counterpart of Problem 2.4. For that purpose, an idealized situation where the porous medium consists of periodically distributed grains and pores is considered. We assume the period is of scale $\varepsilon>0$, and derive the limit problem for $\varepsilon \rightarrow 0$. This limit problem is used as an approximation to the Problem 2.4 for situations with small but non-vanishing scale $\varepsilon$. It bridges the gap between the pore-scale and the scale of the diffusion process.

\subsection{The perforated spatial domain}

Let $\varepsilon>0$ be a small positive parameter. The spatial domain $\Omega \subset \mathbb{R}^{d}, d \in\{2,3\}$, with boundary $\Gamma_{\Omega}=\partial \Omega$, includes no longer only one perforation but many. The grains - these perforations - are small of scale $\varepsilon$. Therefore, the ratio of the scale of the microstructure and the scale of the diffusion process is of order $\varepsilon$.

The grains are periodically distributed with a period of scale $\varepsilon$. In order to model such a situation, it is assumed that $\Omega$ is a finite union of cubes with edge-length $\varepsilon$. Thereto, let $Y$ be a unit cell of dimension $d$ with volume $1-$ for simplicity it is assumed that $Y=(0,1)^{d}$ holds - and let $W_{\Omega}$ be the unique subset of $\mathbb{Z}^{d}$ that satisfies

$$
\Omega=\cup_{w \in W_{\Omega}}\{\varepsilon(w+Y)\} .
$$

Let $n_{\Omega}$ denote the outer-normal to $\Gamma_{\Omega}$. The unit cube $Y$ is subdivided into two regions, the grain $\mathscr{G}$, located in the center of $Y$, and the pore-space $\mathscr{P}$. The grain-boundary $\Gamma_{\mathscr{G}}$ separates $\mathscr{G}$ and $\mathscr{P}$ and $\partial Y$ is the outer boundary of $Y$, that is

$$
Y=\mathscr{G} \cup \Gamma_{\mathscr{G}} \cup \mathscr{P} .
$$

Let $n_{\mathscr{G}}$ be the normal to $\Gamma_{\mathscr{G}}$ that points into the grain. The total pore-space $\mathscr{P}^{\varepsilon}$ of the porous medium is given by

$$
\mathscr{P}^{\varepsilon}=\cup_{w \in W_{\Omega}}\{\varepsilon(w+\mathscr{P})\} .
$$

Its outer-boundary coincides with the boundary $\Gamma_{\Omega}$ of $\Omega$, whereas its inner-boundaries coincide with the total grain surface, that is

$$
\Gamma_{\mathscr{P} \varepsilon}=\Gamma_{\Omega} \cup\left(\cup_{w \in W_{\Omega}}\left\{\varepsilon\left(w+\Gamma_{\mathscr{G}}\right)\right\}\right) .
$$

Let $n \mathscr{P} \varepsilon$ denote the outer normal to $\Gamma_{\mathscr{P} \varepsilon}$.

\subsection{The sequence of problems with varying scale $\varepsilon$}

Define

$$
G_{\varepsilon, i}(\Phi, u)=\left(\sum_{j \in I \backslash\{i\}}\left(\partial_{\phi_{i}}-\partial_{\phi_{j}}\right) W(\Phi)\right)+\varepsilon f_{i}(\Phi, u) \text { for } i \in I .
$$

The scaling of $f_{i}$ is explained below.

On scale $\varepsilon$ the following variant of Problem 2.4 needs to be solved.

Problem 3.1 Given the fixed scale $\varepsilon>0$, the parameters $\alpha, \xi, D, \delta, \rho>0$, the functions $G_{\varepsilon, i}(\Phi, u)$ defined by (3.5), the potential $W(\Phi)$ defined by (2.10), the driving forces $f_{i}(\Phi, u)$ defined by (2.17) and (2.20), the interpolation-function $g_{\delta}(\Phi)$ defined by (2.28), and the periodic initial conditions $\Phi_{\varepsilon, 0}=\left(\phi_{\varepsilon, 1,0}, \phi_{\varepsilon, 2,0}, \phi_{\varepsilon, 3,0}\right): \mathscr{P}^{\varepsilon} \rightarrow \mathscr{S}$ and $u_{\varepsilon, 0}: \mathscr{P}^{\varepsilon} \rightarrow \mathbb{R}$, find the phase-fields $\Phi_{\varepsilon}=\left(\phi_{1, \varepsilon}, \phi_{2, \varepsilon}, \phi_{3, \varepsilon}\right)$ and the concentration $u_{\varepsilon}$ satisfying

$$
\begin{array}{lr}
\alpha \xi^{2} \partial_{t} \phi_{i, \varepsilon}-\frac{3}{4} \xi^{2} \Delta \phi_{i, \varepsilon}+G_{\varepsilon, i}\left(\Phi_{\varepsilon}, u_{\varepsilon}\right)=0 & \text { in } J(T) \times \mathscr{P}^{\varepsilon}, \\
\partial_{t}\left(\left(\phi_{1, \varepsilon}+\delta\right) u_{\varepsilon}\right)-D \nabla \cdot\left(\left(\phi_{1, \varepsilon}+\delta\right) \nabla u_{\varepsilon}\right)-\rho g_{\delta}\left(\Phi_{\varepsilon}\right) \partial_{t} \phi_{1, \varepsilon}= & \text { in } J(T) \times \mathscr{P}^{\varepsilon}, \\
-\frac{3}{4} \xi^{2} \nabla \phi_{i, \varepsilon} \cdot n_{\mathscr{P} \varepsilon}=0 & \text { on } J(T) \times \Gamma_{\mathscr{P} \varepsilon}, \\
-D\left(\phi_{1, \varepsilon}+\delta\right) \nabla u_{\varepsilon} \cdot n_{\mathscr{P} \varepsilon}=0 & \text { on } J(T) \times \Gamma_{\mathscr{P} \varepsilon}, \\
\Phi_{\varepsilon}(0, x)=\Phi_{\varepsilon, 0}(x) & \text { in } \mathscr{P}^{\varepsilon}, \\
u_{\varepsilon}(0, x)=u_{\varepsilon, 0}(x) & \text { in } \mathscr{P}^{\varepsilon} .
\end{array}
$$


Let $C_{\#}^{0}(Y)$ denote the set that contains all periodic continuous functions in $\mathbb{R}^{d}$ with periodicity cell $Y$. The periodicity of the initial data is generated by the conditions

$$
\phi_{\varepsilon, i, 0}(x)=\phi_{0, i, 0}\left(x, \frac{x}{\varepsilon}\right) \text { for } i \in I \text { and } u_{\varepsilon, 0}(x)=u_{0,0}\left(x, \frac{x}{\varepsilon}\right)
$$

with functions $\phi_{0, i, 0}, u_{0,0} \in L_{2}\left(\mathscr{P}^{\varepsilon} ; C_{\#}^{0}(Y)\right)$.

In order to obtain a suitable asymptotic limit problem for $\varepsilon \rightarrow 0$, it is fundamental to scale certain parameters in dependence of $\varepsilon$. Let

$$
\xi=\varepsilon \xi_{0} \text { and } \alpha=\varepsilon^{-2} \alpha_{0} \text { with } \xi_{0}, \alpha_{0}>0 .
$$

Since the volume of a grain is proportional to $\varepsilon^{d}$, the volumes of the surrounding phases are proportional to $\varepsilon^{d}$ too. Consequently, the width $\xi$ of the diffuse interface regions must be proportional to $\varepsilon$. The relaxation parameter $\alpha$ is scaled such that the product $\alpha \xi^{2}$ remains constant. Recalling (2.17), (2.20) and (3.5), the functions $f_{1}\left(\Phi_{\varepsilon}, u_{\varepsilon}\right)$ and $f_{3}\left(\Phi_{\varepsilon}, u_{\varepsilon}\right)$ appearing in the first equation of (3.6) are scaled by $\varepsilon$ such that

$$
\varepsilon f_{1}\left(\Phi_{\varepsilon}, u_{\varepsilon}\right)=-\varepsilon f_{3}\left(\Phi_{\varepsilon}, u_{\varepsilon}\right)=\varepsilon \alpha \xi \gamma_{13}\left(\Phi_{\varepsilon}\right) f\left(u_{\varepsilon}\right)=\alpha_{0} \xi_{0} \gamma_{13}\left(\Phi_{\varepsilon}\right) f\left(u_{\varepsilon}\right) .
$$

The function $f_{2}\left(\Phi_{\varepsilon}, u_{\varepsilon}\right)=0$.

\subsection{The formal asymptotic expansions}

Let $\left(\Phi_{\varepsilon}, u_{\varepsilon}\right)=\left(\phi_{1, \varepsilon}, \phi_{2, \varepsilon}, \phi_{3, \varepsilon}, u_{\varepsilon}\right)$ be the unique solution to the Problem 3.1 of scale $\varepsilon$. In order to study the limit $\varepsilon \rightarrow 0$, the existence of asymptotic expansions

$$
\begin{aligned}
& \phi_{i, \varepsilon}(t, x)=\sum_{j=0}^{\infty} \varepsilon^{j} \phi_{i, j}\left(t, x, \frac{x}{\varepsilon}\right) \text { for } i \in I, \\
& u_{\mathcal{\varepsilon}}(t, x)=\sum_{j=0}^{\infty} \varepsilon^{j} u_{j}\left(t, x, \frac{x}{\varepsilon}\right)
\end{aligned}
$$

of the solution is assumed. Note that the existence of such an expansion is not guaranteed. It is a part of future research to justify the assumption.

Employing the asymptotic expansions, Taylor expansions with the expansion point $\left(\phi_{1,0}, \phi_{2,0}, \phi_{3,0}, u_{0}\right)$ for the functions depending on the solution $\left(\phi_{1, \varepsilon}, \phi_{2, \varepsilon}, \phi_{3, \varepsilon}, u_{\varepsilon}\right)$, as well as the formal relation

$$
\nabla v\left(x, \frac{x}{\varepsilon}\right)=\nabla_{x} v\left(x, \frac{x}{\varepsilon}\right)+\frac{1}{\varepsilon} \nabla_{y} v\left(x, \frac{x}{\varepsilon}\right),
$$

where $\nabla_{x} v$ and $\nabla_{y} v$ denote the gradients with respect to the first and the second variable of a function $v=v(x, y)$, into (3.6) on the scale $\varepsilon$, comparing the coefficients of different powers of $\varepsilon$ and letting the scale parameter $\varepsilon$ tend to 0 , results in the following problems on the different scales.

\subsubsection{The $\varepsilon^{-2}$-problem and the $\varepsilon^{-1}$-condition on the boundary}

The $\mathscr{O}\left(\varepsilon^{-2}\right)$-terms in the evolution equations and the $\mathscr{O}\left(\varepsilon^{-1}\right)$-terms in the boundary conditions of (3.6) give

$$
\begin{aligned}
& -D \nabla_{y} \cdot\left(\left(\phi_{1,0}+\delta\right) \nabla_{y} u_{0}\right)=0 \text { in } J(T) \times \Omega \times \mathscr{P}, \\
& -D\left(\phi_{1,0}+\delta\right) \nabla_{y} u_{0} \cdot n \mathscr{G}=0 \quad \text { on } J(T) \times \Omega \times \Gamma_{\mathscr{G}},
\end{aligned}
$$

where $u_{0}$ is $Y$-periodic with respect to $y$. This implies that the solution $u_{0}=u_{0}(t, x)$ is independent of $y$.

\subsubsection{The $\varepsilon^{-1}$-problem and the $\varepsilon^{0}$-condition on the boundary}

Since $u_{0}=u_{0}(t, x)$, the $\mathscr{O}\left(\varepsilon^{-1}\right)$-terms in the evolution equations and the $\mathscr{O}\left(\varepsilon^{0}\right)$-terms in the boundary conditions of (3.6) give

$$
\begin{aligned}
& -D \nabla_{y} \cdot\left(\left(\phi_{1,0}+\delta\right) \nabla_{y} u_{1}\right)=D \nabla_{y}\left(\phi_{1,0}+\delta\right) \cdot \nabla_{x} u_{0} \text { in } J(T) \times \Omega \times \mathscr{P}, \\
& -D\left(\phi_{1,0}+\delta\right) \nabla_{y} u_{1} \cdot n_{\mathscr{G}}=D\left(\phi_{1,0}+\delta\right) \nabla_{x} u_{0} \cdot n_{\mathscr{G}} \quad \text { on } J(T) \times \Omega \times \Gamma_{\mathscr{G}},
\end{aligned}
$$


where $u_{1}$ is $Y$-periodic with respect to $y$. Because of the linear structure of the problem, the solution $u_{1}$ can be expressed in terms of $\partial_{x_{j}} u_{0}$, that is

$$
u_{1}(t, x, y)=\tilde{u}_{1}(t, x)+\sum_{j=1}^{d} w_{j}(t, x, y) \partial_{x_{j}} u_{0}(t, x)
$$

with an arbitrary function $\tilde{u}_{1}(t, x)$ which plays no role in the final form of the limit problem, and with the unique solutions $w_{j}$ of the cell problems (for $j=1, \ldots, d$ )

$$
\begin{aligned}
& -D \nabla_{y} \cdot\left(\left(\phi_{1,0}+\delta\right) \nabla_{y} w_{j}\right)=D \nabla_{y}\left(\phi_{1,0}+\delta\right) \cdot e_{j} \text { in } J(T) \times \Omega \times \mathscr{P}, \\
& -D\left(\phi_{1,0}+\delta\right) \nabla_{y} w_{j} \cdot n_{\mathscr{G}}=D\left(\phi_{1,0}+\delta\right) e_{j} \cdot n_{\mathscr{G}} \quad \text { on } J(T) \times \Omega \times \Gamma_{\mathscr{G}},
\end{aligned}
$$

where $w_{j}$ is $Y$-periodic with respect to $y$ and its average in $\mathscr{P}$ vanishes, and $e_{j}$ is the $j$-th unit vector of $\mathbb{R}^{d}$.

\subsubsection{The $\varepsilon^{0}$-problem and the $\varepsilon^{1}$-condition on the boundary}

The $\mathscr{O}\left(\varepsilon^{0}\right)$-terms in the evolution equations and the $\mathscr{O}\left(\varepsilon^{1}\right)$-terms in the boundary conditions of (3.6) give

$$
\begin{aligned}
& \alpha_{0} \xi_{0}^{2} \partial_{t} \phi_{i, 0}-\frac{3}{4} \xi_{0}^{2} \Delta_{y} \phi_{i, 0}+\left(\sum_{j \in I \backslash\{i\}}\left(\partial_{\phi_{i}}-\partial_{\phi_{j}}\right) W\left(\Phi_{0}\right)\right) \\
& =-\tilde{f}_{i}\left(\Phi_{0}, u_{0}\right)=-\left\{\begin{array}{ll}
\alpha_{0} \xi_{0} \gamma_{13}\left(\Phi_{0}\right) f\left(u_{0}\right) & \text { if } i=1, \\
0 & \text { if } i=2, \\
-\alpha_{0} \xi_{0} \gamma_{13}\left(\Phi_{0}\right) f\left(u_{0}\right) & \text { if } i=3,
\end{array} \quad \text { in } J(T) \times \Omega \times \mathscr{P},\right. \\
& \partial_{t}\left(\left(\phi_{1,0}+\delta\right) u_{0}\right)-\rho g_{\delta}\left(\Phi_{0}\right) \partial_{t} \phi_{1,0}- \\
& -D \nabla_{x} \cdot\left(\left(\phi_{1,0}+\delta\right)\left(\nabla_{x} u_{0}+\nabla_{y} u_{1}\right)\right)-D \nabla_{y} \cdot \mathscr{A}=0 \quad \text { in } J(T) \times \Omega \times \mathscr{P} \text {, } \\
& -\frac{3}{4} \xi_{0}^{2} \nabla_{y} \phi_{i, 0} \cdot n_{\mathscr{G}}=0 \quad \text { on } J(T) \times \Omega \times \Gamma_{\mathscr{G}} \text {, } \\
& -D \mathscr{A} \cdot n_{\mathscr{G}}=0 \quad \text { on } J(T) \times \Omega \times \Gamma_{\mathscr{G}} \text {, }
\end{aligned}
$$

where

$$
\mathscr{A}=\left(\phi_{1,0}+\delta\right)\left(\nabla_{x} u_{1}+\nabla_{y} u_{2}\right)+\phi_{1,1}\left(\nabla_{x} u_{0}+\nabla_{y} u_{1}\right),
$$

and where $\phi_{i, 0}$ and $u_{2}$ are $Y$-periodic with respect to $y$. In order to dispose of the term $-D \nabla_{y} \cdot \mathscr{A}$ in the second evolution equation, the equation is integrated over the domain $\mathscr{P}$. Since $u_{0}$ is independent of $y$, the periodicity of $\phi_{1,0}, \phi_{1,1}, u_{1}$ and $u_{2}$ yields the homogenized equation

$$
\partial_{t}\left({\overline{\phi_{1,0}+\delta}}^{\mathscr{P}} u_{0}\right)-D \nabla_{x} \cdot\left({\overline{K\left(\phi_{1,0}+\delta, w_{1}, w_{2}\right)}}^{\mathscr{P}} \nabla_{x} u_{0}\right)-\rho{\overline{g_{\delta}\left(\Phi_{0}\right) \partial_{t} \phi_{1,0}}}^{\mathscr{P}}=0
$$

in $J(T) \times \Omega$, where for all functions $v: \overline{J(T)} \times \Omega \times \mathscr{P} \rightarrow \mathbb{R}$

$$
\overline{v(t, x, \cdot)}^{\mathscr{P}}=\frac{1}{|\mathscr{P}|} \int_{\mathscr{P}} v(t, x, y) d y
$$

and

$$
\left(\overline{K_{\left(\phi_{1,0}+\delta, w_{1}, w_{2}\right)(t, x, \cdot)}^{\mathscr{P}}}\right)_{i j}:=\frac{1}{|\mathscr{P}|} \int_{\mathscr{P}}\left(\left(\phi_{1,0}+\delta\right)\left(\delta_{i j}+\partial_{y_{i}} w_{j}\right)\right)(t, x, y) d y \text { for } 1 \leqslant i, j \leqslant d .
$$

REMARK 3.1 In Hornung (1997) it is proven that the effective diffusion tensor $\overline{K\left(\phi_{1,0}+\delta, w_{1}, w_{2}\right)}$ is symmetric, elliptic and bounded.

\subsection{The two-scale model}

The limit problem is a two-scale one, which is used as approximation of the pore-scale Problem 2.4 for situations with small but non-vanishing scale parameter $\varepsilon$. It bridges the gap between the pore-scale and the scale of the diffusion process. For ease of the presentation the subscript 0 referring to the scale $\varepsilon^{0}$ is omitted in the following. The two-scale model consists of a macroscopic homogenized equation that describes the evolution of the macroscopic concentration 


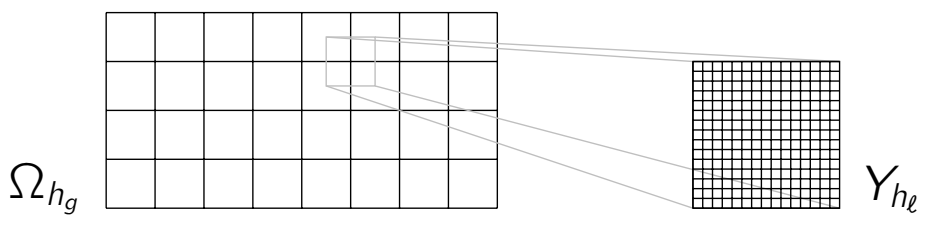

FIG. 3.1: The macroscopic $\Omega_{h_{g}}$ and the local microscopic grid $Y_{h_{\ell}}$.

$u=u(t, x)$ of the dissolved particles in the fluid $P_{1}$, and at each point of the macroscopic domain of microscopic cell problems with periodic boundary conditions that describe the evolution of the microscopic phase-fields $\Phi=\Phi(t, x, y)$ and of the microscopic auxiliary functions $w_{1}=w_{1}(t, x, y)$ and $w_{2}=w_{2}(t, x, y)$. The variables on the two scales are coupled: the macroscopic equation depends on averaged values of the solution of the local cell problems and, vice versa, the macroscopic concentration appears in the driving forces of the phase-field equations in the local cell problems. What is important to note is that the microscopic problems in different macroscopic nodes do not influence each other directly. Therefore, they can be computed in parallel.

The macroscopic problem and the family of microscopic cell problems have the following formulation.

Problem 3.2 Given the parameters $\alpha, \xi, D, \delta, \rho>0$, the functions $G_{i}(\Phi, u)$ defined by (2.21), the potential $W(\Phi)$ defined by (2.10), the driving forces $f_{i}(\Phi, u)$ defined by (2.17) and (2.20), the interpolation-function $g_{\delta}(\Phi)$ defined by (2.28), the averaging $\bar{u}^{\mathscr{P}}$ of functions $u: \overline{J(T)} \times \Omega \times \mathscr{P} \rightarrow \mathbb{R}$ defined by (3.19), the effective diffusion coefficient ${\overline{K\left(\phi_{1}+\delta, w_{1}, w_{2}\right)}}^{\mathscr{P}}$ defined by (3.20), and the initial conditions $\Phi_{0}=\left(\phi_{1,0}, \phi_{2,0}, \phi_{3,0}\right): \Omega \times \mathscr{P} \rightarrow \mathscr{S}$ and $u_{0}: \Omega \rightarrow \mathbb{R}$. Find the macroscopic concentration $u$ satisfying

$$
\begin{array}{lc}
\partial_{t}\left({\overline{\phi_{1}+\delta}}^{\mathscr{P}} u\right)-D \nabla_{x} \cdot\left({\overline{K\left(\phi_{1}+\delta, w_{1}, w_{2}\right)}}^{\mathscr{P}} \nabla_{x} u\right)-\rho{\overline{g_{\delta}(\Phi) \partial_{t} \phi_{1}}}^{\mathscr{P}}=0 & \text { in } J(T) \times \Omega, \\
-D \overline{K\left(\phi_{1}+\delta, w_{1}, w_{2}\right)}{ }^{\mathscr{P}} \nabla_{x} u \cdot n_{\Omega}=0 & \text { on } J(T) \times \Gamma_{\Omega} . \\
u(0, x)=u_{0}(x) & \text { in } \Omega .
\end{array}
$$

For every $x \in \Omega$ find the $Y$-periodic microscopic phase-fields $\Phi$ satisfying for $i \in I$

$$
\begin{array}{lr}
\alpha \xi^{2} \partial_{t} \phi_{i}-\frac{3}{4} \xi^{2} \Delta_{y} \phi_{i}+G_{i}(\Phi, u)=0 \text { in } J(T) \times \mathscr{P}, \\
-\frac{3}{4} \xi^{2} \nabla_{y} \phi_{i} \cdot n_{\mathscr{G}}=0 & \text { on } J(T) \times \Gamma_{\mathscr{G}} .
\end{array}
$$

For every $x \in \Omega$ and for $j=1, \ldots, d$ find the $Y$-periodic microscopic solution $w_{j}$ with vanishing average in the porespace $\mathscr{P}$ satisfying

$$
\begin{aligned}
& -D \nabla_{y} \cdot\left(\left(\phi_{1}+\delta\right) \nabla_{y} w_{j}\right)=D \nabla_{y}\left(\phi_{1}+\delta\right) \cdot e_{j} \text { in } J(T) \times \mathscr{P} \\
& -D\left(\phi_{1}+\delta\right) \nabla_{y} w_{j} \cdot n_{\mathscr{G}}=D\left(\phi_{1}+\delta\right) e_{j} \cdot n_{\mathscr{G}} \quad \text { on } J(T) \times \Gamma_{\mathscr{G}} .
\end{aligned}
$$

\subsection{An adaptive discretization of the two-scale problem}

Problem 3.2 is discretized explicitly in time. The spatial derivatives are discretized by standard second-order finite differences on uniform rectangular grids $\Omega_{h_{g}}$ and $Y_{h_{\ell}}$, where $h_{g}>0$ denotes the macroscopic and $h_{\ell}>0$ the microscopic space-increment. The discrete pore-space $\mathscr{P}_{h_{\ell}}$ as well as the physical and numerical constants are specified in the Section 4 presenting the results of numerical computations. Due to the CFL-condition, the time-increment $\Delta t=\Delta^{n} t$ must satisfy

$$
\Delta^{n} t \leqslant c(D)\left(\delta+\min _{x \in \Omega_{h g}}{\overline{\phi_{1}\left(t_{n-1}, x\right)}}^{\mathscr{P}_{h_{\ell}}}\right) h_{g}^{2}
$$

where $t_{n}$ denotes the discrete time in step $n$ and the constant $c(D)>0$ depends on the diffusion coefficient $D$. The Figure 3.1 illustrates the macro-micro configuration.

It is demanding to compute the microscopic parameters for the macroscopic problem in every grid point of the macroscopic domain. The solution of a microscopic problem depends continuously on the evolution of the macroscopic 
concentration. Exploiting this property, the two-scale model is solved adaptively in the sense of the strategy introduced in Redeker $\&$ Eck (2013). A metric $d_{E}$ measures the distance of two concentration evolutions $u(\cdot, x)$ and $u(\cdot, \tilde{x})$ on the time-interval $\overline{J(T)}$ in two macroscopic nodes $x, \tilde{x} \in \Omega_{h_{g}}$,

$$
d_{E}(u(\cdot, x), u(\cdot, \tilde{x}) ; t, \lambda):=\int_{0}^{t} e^{-\lambda(t-s)}|u(s, x)-u(s, \tilde{x})| d s,
$$

where $\lambda \geqslant 0$ is a history parameter. A microscopic problem is solved for active macroscopic nodes only, the microscopic data of inactive macroscopic nodes is interpolated between the microscopic data of active nodes. Initially, the set of active nodes contains only one macroscopic node. It is updated in course of the simulation. That is, it is refined if necessary, that is if the minimal $d_{E}$-distance between an inactive and all active nodes exceeds an upper bound. Vice versa, it is coarsened if possible, that is if the $d_{E}$-distance between two active nodes falls below a lower limit. The interested reader is referred to Redeker \& Eck (2013) for more details on the strategy.

The following algorithm solves the adaptive discrete problem.

1. Set $n=n+1$ and $t_{n}=t_{n-1}+\Delta^{n} t$.

2. Adjust the set $A^{n}$ of the active macroscopic nodes.

3. For every node in $A^{n}$ solve (3.22) for $\phi_{2}^{n}$ and $\phi_{3}^{n}$ and set $\phi_{1}^{n}=1-\phi_{2}^{n}-\phi_{3}^{n}$.

4. For every node in $A^{n}$ solve (3.23) for $w_{1}^{n}$ and $w_{2}^{n}$.

5. Approximate the microscopic parameters ${\overline{\phi_{1}+\delta}}^{\mathscr{P}},{\overline{K\left(\phi_{1}+\delta, w_{1}, w_{2}\right)}}^{\mathscr{P}}$ and ${\overline{g_{\delta}(\Phi) \partial_{t} \phi_{1}}}^{\mathscr{P}}$ in $\Omega_{h_{g}}$.

6. Solve the macroscopic problem (3.21) for $u^{n}$.

7. Restart if $t_{n}<T$.

Fundamental to the adaptive strategy is that the initial conditions of the microscopic problems are at least very similar. Therefore, as it is in some of the settings considered in the Section 4, if these initial conditions differ, then the set of macroscopic nodes must be subdivided into subsets with respect to these initial conditions. That is, for $M$ initial conditions of the microscopic problems $M$ subsets $\Omega_{h_{g}}^{(m)}, m=1, \ldots, M$, of macroscopic nodes $\Omega_{h_{g}}$ are constructed. For each of the subsets one adaptive solution strategy is applied for.

For completeness, the parameters of the adaptive strategy are specified as follows, see Redeker \& Eck (2013): $\lambda=0.1, c_{\mathrm{tol}_{\mathrm{c}}}=0.2, c_{\mathrm{tol}_{\mathrm{e}}}=0.01, \mathrm{RU}=0, e C=1, M_{A}=2$, IT-CA, the initial set of active nodes contains one node of every subset $\Omega_{h_{g}}^{(m)}$, the Copy Method, and the refining tolerance is defined as

$$
\operatorname{tol}_{\mathrm{r}}(t):=c_{\mathrm{tol}_{\mathrm{r}}} \max _{m=1, \ldots, M} \max _{x, \tilde{x} \in \Omega_{h_{g}}^{(m)}}\left\{d_{E}(u(\cdot, x), u(\cdot, \tilde{x}) ; t, \lambda)\right\},
$$

where $c_{\mathrm{tol}_{\mathrm{r}}}=10^{-3 / 2}$ if not stated otherwise.

REMARK 3.2 In case of the initial configurations $\mathscr{I}_{0}$ and $\mathscr{I}_{2}$ it is not necessary to solve (3.23) for both $w_{1}^{n}$ and $w_{2}^{n}$, since they satisfy $w_{2}^{n}\left(y_{0}, y_{1}\right)=w_{1}^{n}\left(y_{1}, y_{0}\right)$ for all $y=\left(y_{0}, y_{1}\right) \in \mathscr{P}$. Due to the fact that the complexity of the discrete (3.23) is high (and significantly higher than of (3.22)), the application of Model Reduction techniques to (3.23) is appropriate and accelerates the computation time significantly. For a related application Reduced Basis methods have been suggested in Redeker \& Haasdonk (2014).

\section{Numerical results}

In this section we present numerical solutions of the two-scale Problem 3.2 in two spatial dimensions which are obtained with the method from Section 3.5. We begin with validation examples of the numerical approach that include a test on the temporal behaviour of the free energy $U(\Phi)$ in the microscopic pore-space (Test $E$, Section 4.1 ) and on the accuracy of the adaptive strategy (Test $A$, Section 4.2).

The major contributions of this section are contained in Section 4.3 with five application examples denoted by Tests $S_{1}, \ldots, S_{5}$. They display in particular the interdependence of the change of the pore structure and the evolution of the macroscopic concentration of the dissolved particles in the fluid $P_{1}$.

Note that, for ease of presentation, the distinction between for example water $P_{1}$ and the diffuse domain $P_{1}^{d}(t)$ occupied by water is no longer made. Therefore, $P_{1}$ refers either to water or to the occupied domain.

The different parameters in the Tests $E, A, S_{1}, \ldots, S_{5}$ are specified in Table 4.1. The remaining common parameters are specified in the text following. The interval $[0, T]$ is partitioned using the time-dependent increments $\Delta^{n} t=$ $0.001\left(\delta+\min _{x \in \Omega_{h_{g}}}{\overline{\phi_{1}\left(t_{n-1}, x\right)}}_{h_{\ell}}^{\mathscr{P}_{h}}\right), \delta=1 e-6$. The microscopic domain $Y_{h_{\ell}}$ is perforated by a small spherical grain $\mathscr{G}_{h_{\ell}}$ located in the center of $Y_{h_{\ell}}$ whose radius equates the microscopic space increment $h_{\ell}=0.1$. Let $H_{\ell}$ denote the 


\begin{tabular}{c|c|cccc|cccccc} 
test & $T$ & $H_{g}$ & $u_{0}$ & $D$ & $u_{\perp}(t)$ & $H_{\ell}$ & $\mathscr{I}$ & $\tilde{r}_{0}$ & $M$ & $r_{0}$ & $c_{u, 0}$ \\
\hline$E$ & 15 & & & & & 200 & $\mathscr{I}_{1}$ & - & 2 & $\{20,80\}$ & 0 \\
$A$ & 9 & $\{9,9\}$ & 0.4 & $5 e+2$ & $0.8: 0.8$ & 100 & $\mathscr{I}_{2}$ & 20 & 2 & $\{10,30\}$ & 100 \\
$S_{1}$ & 57 & $\{50,25\}$ & 0.5 & $2 e+4$ & $0.5: 0.9$ & 200 & $\mathscr{I}_{0}$ & - & 1 & 30 & 100 \\
$S_{2}$ & 51 & & 0.5 & & $0.5: 0.82$ & & $\mathscr{I}_{2}$ & 30 & 1 & 30 & \\
$S_{3}$ & 32 & & 0.5 & & $0.5: 0.9$ & & $\mathscr{I}_{2}$ & 30 & 2 & $\{30,70\}$ & \\
$S_{4}$ & 23 & & 0.5 & & $0.5: 0.9$ & & $\mathscr{I}_{1}$ & - & 2 & $\{30,70\}$ & \\
$S_{5}$ & 44 & & 0.35 & & $0.4: 0.9$ & & $\mathscr{I}_{2}$ & 30 & 2 & $\{30,70\}$
\end{tabular}

Table 4.1: The varying parameters of the different tests. Since only the microscopic problems are considered in Test $E$, the macroscopic parameters are not specified. The five Tests $S_{1}, \ldots, S_{5}$ differ from each other only in the parameters that are specified for all of them. The function $u_{\perp}(t)=a: b$ increases monotonically from $a$ to $b$ in course of a simulation.

number of the microscopic nodes in both space dimensions. Then, the microscopic domain $Y_{h_{\ell}}$ and the pore-space $\mathscr{P}_{h_{\ell}}$ are given by

$$
Y_{h_{\ell}}=\left\{y_{h_{\ell}}=\left(k_{1} h_{\ell}, k_{2} h_{\ell}\right) \mid k_{1}, k_{2} \in\left\{0,1, \ldots, H_{\ell}\right\}\right\}, \mathscr{P}_{h_{\ell}}=Y_{h_{\ell}} \backslash \mathscr{G}_{h_{\ell}} .
$$

The pore-space $\mathscr{P}_{h_{\ell}}$ consists of only $H_{\ell}^{2}-1$ nodes, due to the periodic boundary conditions and the grain. The edgelength $h_{g}=H_{\ell} h_{\ell}$ of $Y_{h_{\ell}}$ defines the macroscopic space-increment $h_{g}$. The macroscopic domain $\Omega_{h_{g}}$,

$$
\Omega_{h_{g}}=\left\{x_{h_{g}}=\left(k_{1} h_{g}, k_{2} h_{g}\right) \mid k_{1} \in\left\{0,1, \ldots, H_{g, 1}\right\}, k_{2} \in\left\{0,1, \ldots, H_{g, 2}\right\}\right\}
$$

consists of $\left(H_{g, 1}+1\right)\left(H_{g, 2}+1\right)$ nodes. The initial concentration $u_{0}$ and the diffusion coefficient $D$ are chosen to be constant. It is assumed that $\Omega$ is surrounded by a virtual domain $\Omega_{V}$, that means that the mixture in $\Omega$ is surrounded by a virtual mixture. For $\left(t, x_{V}\right) \in \overline{J(T)} \times \Omega_{V}$ it is prescribed that

$$
{\overline{K\left(\phi_{1}+\delta, w_{1}, w_{2}\right)\left(x_{V}\right)}}^{\mathscr{P}}=\overline{K\left(\phi_{1}+\delta, w_{1}, w_{2}\right)\left(x_{\perp}\left(x_{V}\right)\right)} \text {, }
$$

where

$$
x_{\perp}\left(x_{V}\right)=\left(x_{\perp, 1}\left(x_{V}\right) \quad x_{\perp, 2}\left(x_{V}\right)\right)^{\top}:=\arg \min _{x \in \Omega}\left|x-x_{V}\right| .
$$

Furthermore, the concentration in $\Omega_{V}$ is prescribed in a way that dissolved particles flow into $\Omega$ across its upper and its right boundary while the remaining part of the boundary is not permeable for the dissolved particles. Precisely, for all $\left(t, x_{V}\right) \in \overline{J(T)} \times \Omega_{V}$ we have

$$
u\left(x_{V}, t\right)= \begin{cases}u_{\perp}(t) & \text { if } x_{\perp, 1}\left(x_{V}\right)=H_{g_{1}} h_{g} \text { or if } x_{\perp, 2}\left(x_{V}\right)=H_{g_{2}} h_{g}, \\ u\left(x_{\perp}\left(x_{V}\right)\right) & \text { elsewhere. }\end{cases}
$$

The other microscopic physical parameters are given by $\alpha=100, \xi=0.25$, and the concentration $\rho$ of particles in the precipitate $P_{3}$ satisfies $\rho \equiv 1$. The parameters of the rate-function $f$ from (2.5) are chosen as $c_{u, 1}=0.5$ and $c_{u, 0}>0$ is constant and specified in Table 4.1 .

It remains to specify the initial configuration of the phase-fields. The grain is surrounded by a spherical precipitate $P_{3}$ as displayed in Figure 4.1. Three initial configurations $\mathscr{I}=\left\{\mathscr{I}_{0}, \mathscr{I}_{1}, \mathscr{I}_{2}\right\}$ are considered. For initial configuration $\mathscr{I}_{0}$ there is only one fluid, $P_{1}$. For initial configuration $\mathscr{I}_{1}$ we have also the fluid $P_{2}$, it occupies the right and $P_{1}$ the left part of the non- $P_{3}$ domain. Finally, for initial configuration $\mathscr{I}_{2}$ the fluid $P_{2}$ is located in the corners of the microscopic domain $Y$. Due to the periodic boundary conditions $P_{2}$ is spherical with radius $\tilde{r}_{0}$. For the parameter $M=1$ all precipitates have the same initial radius $r_{0,1}$. If $M=2$, the domain $\Omega_{h_{g}}$ is subdivided into the two subdomains $\Omega_{h_{g}}^{(1)}=\Omega_{h_{g}} \backslash \Omega_{h_{g}}^{(2)}$ and $\Omega_{h_{g}}^{(2)}$, where

$$
\Omega_{h_{g}}^{(2)}=\left\{x_{h_{g}} \in \Omega_{h_{g}} \mid x_{h_{g}} \in\left[\left\lfloor\frac{H_{g, 1}}{4}\right\rfloor h_{g}, 3\left\lfloor\frac{H_{g, 1}}{4}\right\rfloor h_{g}\right] \times\left[\left\lfloor\frac{H_{g, 2}}{4}\right\rfloor h_{g}, 3\left\lfloor\frac{H_{g, 2}}{4}\right\rfloor h_{g}\right\rfloor\right\},
$$




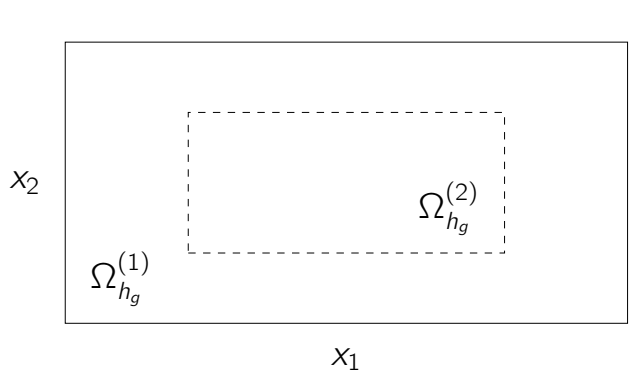

(a) The sub-domains of $\Omega_{h_{g}}$.

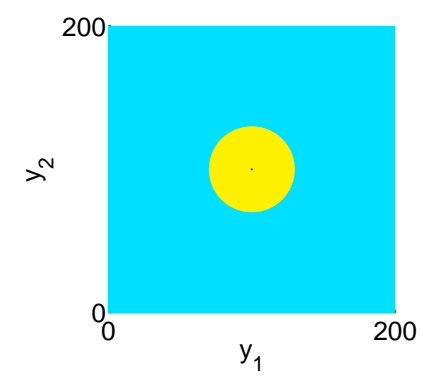

(b) $\mathscr{I}_{0}, \Omega_{h_{g}}^{(1)}: r_{0,1}=30 h_{\ell}$.

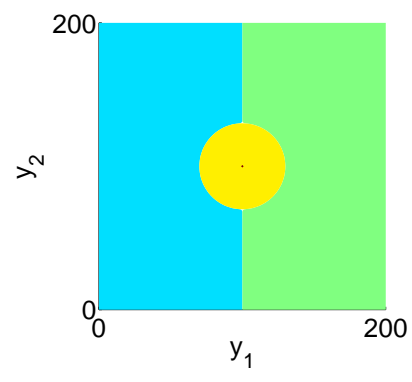

(c) $\mathscr{I}_{1}, \Omega_{h_{g}}^{(1)}: r_{0,1}=30 h_{\ell}$.

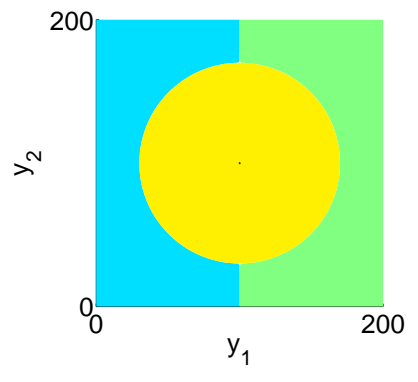

(d) $\mathscr{I}_{1}, \Omega_{h_{g}}^{(2)}: r_{0,2}=70 h_{\ell}$.

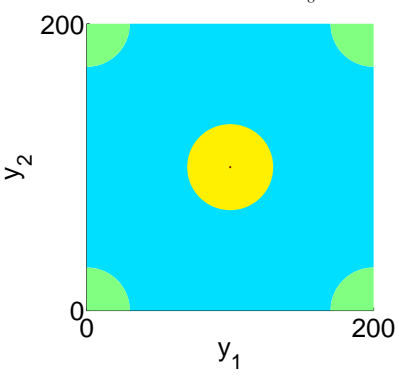

(e) $\mathscr{I}_{2}, \Omega_{h_{g}}^{(1)}: r_{0,1}=30 h_{\ell}$.

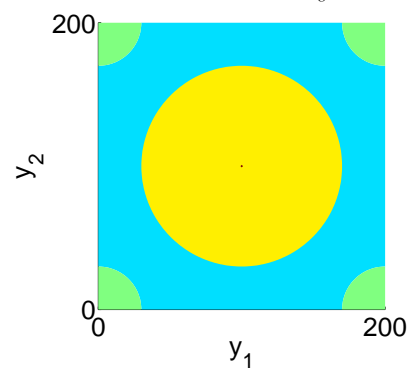

(f) $\mathscr{I}_{2}, \Omega_{h_{g}}^{(2)}: r_{0,2}=70 h_{\ell}$.

FIG. 4.1: The initial phase configurations for the parameter $M=2$ in the microscopic pore-space $\mathscr{P}_{h_{\ell}}$ in the two sub-domains $\Omega_{h_{g}}^{(1)}$ and $\Omega_{h_{g}}^{(2)}$ of the macroscopic domain $\Omega_{h_{g}}$. The black points in the very center of $\mathscr{P}_{h_{\ell}}$ in (b) - (f) represent the small spherical grains $\mathscr{G}_{h_{\ell}}$. Each grain is surrounded by a precipitate $P_{3}$ (yellow). The radii $r_{0,1}<r_{0,2}$ of the initial precipitate domains are constant in both sub-domains $\Omega_{h_{g}}^{(1)}$ and $\Omega_{h_{g}}^{(2)}$. The colored phase domains $P_{i}$ are separated by the level sets $\phi_{i}=\frac{1}{2}$ for $i \in I$.

with the function $x \mapsto\lfloor x\rfloor=\max \{k \in \mathbb{Z} \mid k \leqslant x\}$. Then the precipitates in $\Omega_{h_{g}}^{(1)}$ have initially radius $r_{0,1}$, and in $\Omega_{h_{g}}^{(2)}$ the radius $r_{0,2}>r_{0,1}$. The initial configuration for $M=2$ and for $\mathscr{I}_{1}$ and $\mathscr{I}_{2}$ is illustrated in Figure 4.1. Note that if the fluid $P_{2}$ is not existent initially then $\phi_{2} \equiv 0$ in $J_{\Delta^{n} t}(T) \times \Omega_{h_{g}} \times \mathscr{P}_{h_{\ell}}$. The phase-fields are initialized by $\Phi_{0}=\left(\phi_{1,0}, \phi_{2,0}, \phi_{3,0}\right)$ in accordance with the equation (2.77).

\subsection{Test $E$ : the evolution of the free energy}

Figure 4.2a illustrates for Test $E$ the evolution of the free energy $U(\Phi)$ from (2.9) for the microscopic problems in the two sub-domains $\Omega_{h_{g}}^{(1)}$ and $\Omega_{h_{g}}^{(2)}$ and the initial configuration $\mathscr{I}_{1}$. Note that already initially diffuse triple junctions $\Gamma_{123}$ exist. The driving forces vanish, since $c_{u, 0}=0$ (see (2.5)). In both sub-domains the free energy decreases monotonically in $J_{\Delta^{n} t}(15)$. The rapid decrease in the beginning results from presumably slightly non-compatible initial conditions $\Phi_{0}$; non-compatible in the sense that the initial phase transition width does not fit to the one determined by the evolution. However, the monotone decrease matches the expectation of a dissipative free energy (see Theorem 2.7). Figures 4.2b and $4.2 \mathrm{c}$ show that the initially spherical phase $P_{3}$ converges to its equilibrium state, that takes the shape of a lense between the two fluids. Because the surface tension parameter $\sigma$ is the same in the three phases, the three interfaces meet with an angle of $120^{\circ}$ in equilibrium. This supports the results presented in Section 2.3.

\subsection{Test A: the quality of the adaptive strategy}

The results of Test $A$ are presented in Figure 4.3. They show that the adaptive solution converges to the non-adaptive solution if the tolerance parameter $c_{\mathrm{tol}_{\mathrm{r}}}$ of (3.26) tends to zero. Since the adaptive result converges rapidly to the nonadaptive result, the adaptive results of Test $S_{1}, \ldots, S_{5}$ below are trustworthy. Furthermore, the rapid convergence shows that it is not efficient to compute the microscopic parameters for the macroscopic problem in each macroscopic node. 


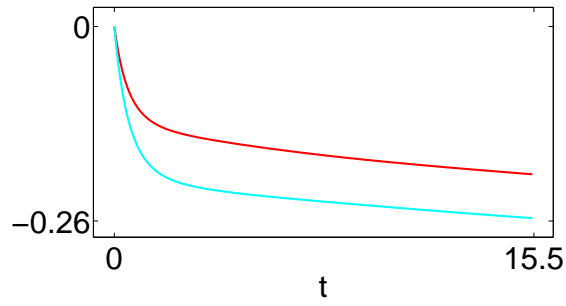

(a) $U(\Phi(t))-U\left(\Phi_{0}\right)$.

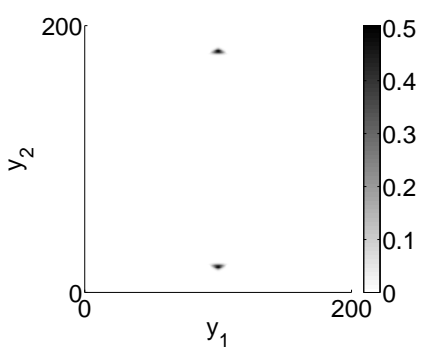

(b) $\left[\phi_{3}(15)-\phi_{3}(0)\right]_{+}$.

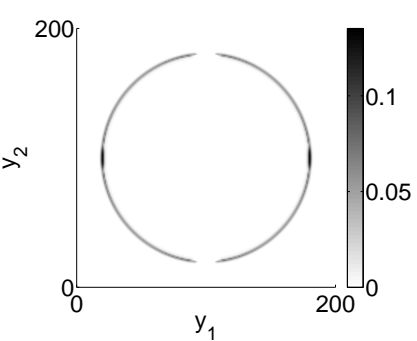

(c) $-\left[\phi_{3}(15)-\phi_{3}(0)\right]_{-}$.

FIG. 4.2: Results of Test $E$ with vanishing driving forces on the time-interval $J_{\Delta^{n} t}(15)$ for initial configuration $\mathscr{I}_{1}$. Figure (a) depicts the evolution of the free energy difference $U(\Phi(t))-U\left(\Phi_{0}\right)$ in dark-gray (resp. light-gray) for the microscopic problems in the sub-domain $\Omega_{h_{g}}^{(1)}$ (resp. $\Omega_{h_{g}}^{(2)}$ ) of the macroscopic domain $\Omega_{h_{g}}$. The initial free energy satisfies $U\left(\Phi_{0}\right)=11.36$ everywhere in $\Omega_{h_{g}}^{(1)}$ and $U\left(\Phi_{0}\right)=17.48$ in $\Omega_{h_{g}}^{(2)}$. The Figures (b) and (c) depict the positive and the negative portion of the difference $\phi_{3}(15)-\phi_{3}(0)$ for the microscopic problems in $\Omega_{h_{g}}^{(2)}$, where $[\cdot]_{+}: x \mapsto[x]_{+}=\max (0, x)$ and $[\cdot]_{-}: x \mapsto[x]_{-}=\min (0, x)$. The precipitate $P_{3}$ converges towards the form of a lense between the two fluids.

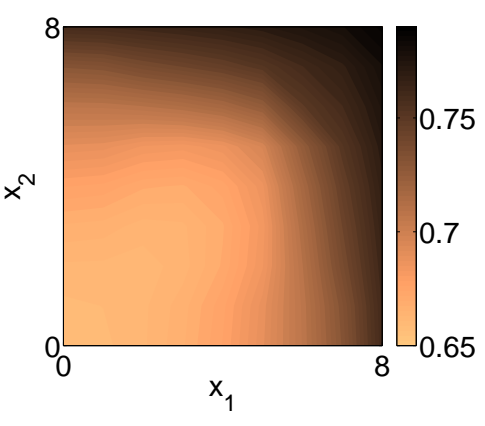

(a) Macroscopic $u$.

\begin{tabular}{c|ccc}
$c_{\text {tol }_{\mathrm{r}}}$ & $\|\Delta u\|_{J \times \Omega, \infty}$ & $\|\Delta u\|_{J \times \Omega, 2}$ & $\overline{\left|N_{A}\right|}$ \\
\hline 1 & $5.64 e-03$ & $2.70 e-03$ & 4.00 \\
$10^{-1 / 2}$ & $2.57 e-02$ & $2.68 e-03$ & 6.33 \\
$10^{-1}$ & $1.60 e-03$ & $2.30 e-04$ & 14.45 \\
$10^{-3 / 2}$ & $6.08 e-04$ & $2.26 e-04$ & 28.84 \\
$10^{-2}$ & $8.59 e-05$ & $3.44 e-05$ & 38.57 \\
0 & $0.00 e+0$ & $0.00 e+0$ & 81.00
\end{tabular}

(b) Convergence for $c_{\mathrm{tol}_{\mathrm{r}}} \rightarrow 0$.

FIG. 4.3: Convergence of the adaptive solution to the non-adaptive solution of Test $A$ for different tolerance parameters $c_{\text {tol }}$ (see (3.26)). Figure (a) depicts the macroscopic concentration of the non-adaptive solution at the end time $T=9$. The second resp. the third column of the table present the $L_{\infty}$ - resp. $L_{2}$-norm of the difference $\Delta u$ of the adaptive and the non-adaptive macroscopic concentration solution in the discrete time-space cylinder $J_{\Delta^{n} t}(9) \times \Omega_{h_{g}}$. In addition, the fourth column gives the average number $\overline{\left|N_{A}\right|}$ of active nodes in $J_{\Delta^{n} t}(9)$.

\subsection{The Tests $S_{1}, S_{2}, S_{3}, S_{4}$ and $S_{5}$}

The Tests $S_{1}, \ldots, S_{5}$ solve the two-scale Problem 3.2 on a large time-space cylinder $J_{\Delta^{n} t}(T) \times \Omega_{h_{g}} \times \mathscr{P}_{h_{\ell}}$. The macroscopic domain $\Omega_{h_{g}}=\left[0,49 h_{g}\right] \times\left[0,24 h_{g}\right]$ contains 1,250 nodes, each of them governs one microscopic problem in $\mathscr{P}_{h_{\ell}}$ consisting of 40,000 nodes. Consequently, the product $\Omega_{h_{g}} \times \mathscr{P}_{h_{\ell}}$ consists of $5 e+7$ nodes. We apply the adaptive strategy with $c_{\mathrm{tol}_{\mathrm{r}}}=10^{-3 / 2}$.

The tests differ concerning the initially constant macroscopic concentration $u_{0}$, the parameter $M$ (specifying whether the macroscopic domain is subdivided into two sub-domains $\Omega_{h_{g}}^{(1)}$ and $\Omega_{h_{g}}^{(2)}$ ), and the initial configuration of the three phases. The initial volume fraction $\overline{\phi_{3,0}} \mathscr{P}$ of the spherical initial precipitate $P_{3}$ of radius $r_{0}=30 h_{\ell}$ equals 0.0707 , and ${\overline{\phi_{3}}}^{\mathscr{P}}=0.3848$ if $r_{0}=70 h_{\ell}$. In case of the initial configuration $\mathscr{I}_{2}$ the fluid phase $P_{2}$ of radius $\tilde{r}_{0}=30 h_{\ell}$ leads to the value $\overline{\phi_{2,0}} \stackrel{\mathscr{P}}{=}=0.0707$. 


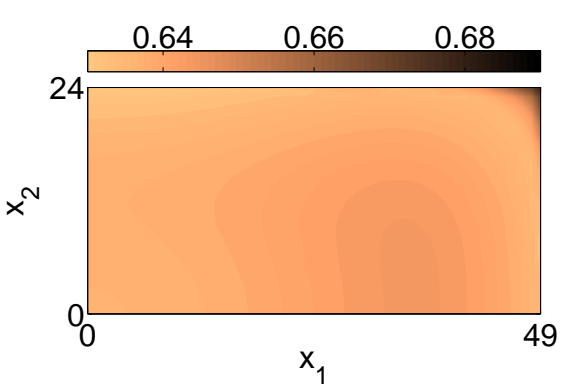

(a) Macroscopic $u$.

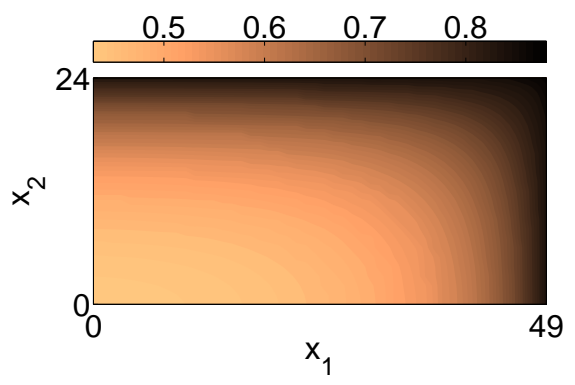

(b) Macroscopic ${\overline{\phi_{3}}}^{\mathscr{P}}$.

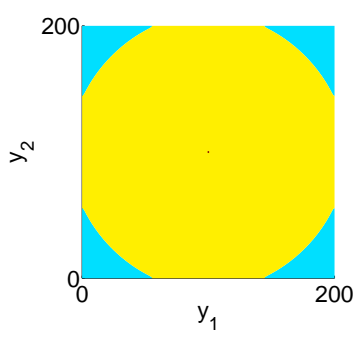

(c) Phases in $(49,24)^{\top}$.

FIG. 4.4: Results of Test $S_{1}$ in the end of the time-interval $J_{\Delta^{n} t}(57)$. The Figure (a) depicts the macroscopic concentration $u$, (b) displays the macroscopic ratio ${\overline{\phi_{3}}}^{\mathscr{P}}$ of the precipitate volume in the local pore-space, and (c) illustrates the configuration of the phases in the pore-space in the macroscopic node $(49,24)^{\top}$. Since the fluid $P_{2}$ is not existent in the beginning and hence at any time, only the fluid $P_{1}$ and the precipitate $P_{3}$ show up.

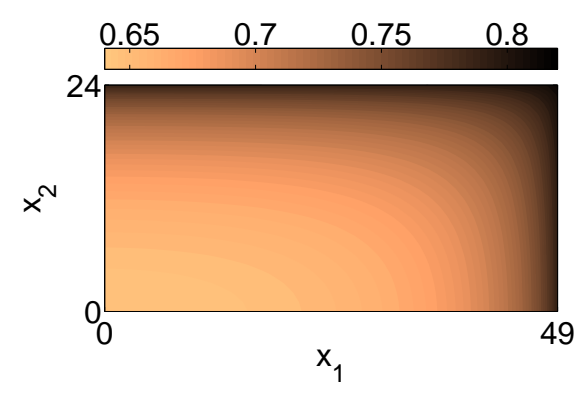

(a) Macroscopic $u$.

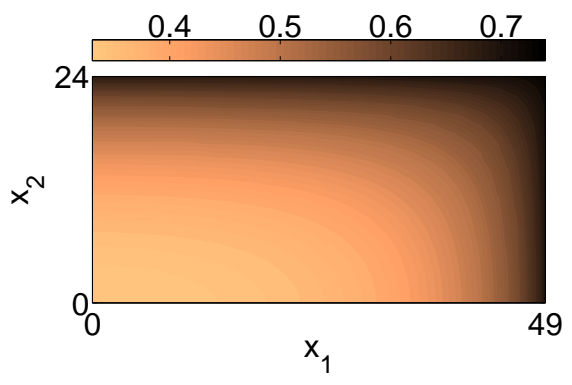

(b) Macroscopic ${\overline{\phi_{3}}}^{\mathscr{P}}$.

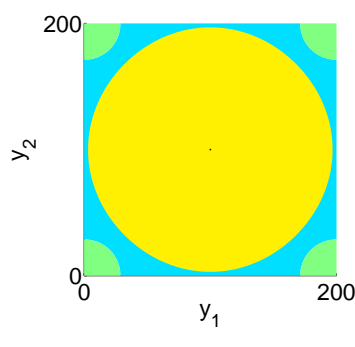

(c) Phases in $(49,24)^{\top}$.

FIG. 4.5: Results of Test $S_{2}$ in the end of $J_{\Delta^{n}}(51)$.

\subsubsection{The Tests $S_{1}$ and $S_{2}$}

Figure 4.4 illustrates the results of Test $S_{1}$ for $\mathscr{I}_{2}$. Due to $M=1$ all initial precipitates are of radius $r_{0,1}=30 h_{\ell}$, that is the initial precipitate volume fraction of the pore-space volume equals 0.0707 . The initial concentration $u_{0}=0.5$ matches the threshold $c_{u, 1}=0.5$ of the rate-function in (2.5). Therefore, the existing phases $P_{1}$ and $P_{3}$ are almost constant for small times, because neither precipitation nor dissolution takes place.

The concentration $u$ is never smaller than the threshold and never larger than 0.9 in $J_{\Delta^{n} t}(57) \times \Omega_{h_{g}}$. Due to the transport of particles into $\Omega_{h_{g}}$ across the right and across the upper boundary (4.5), the macroscopic node $(49,24)^{\top}$ records all the time the highest concentration, and $(0,0)^{\top}$ the lowest. Due to the fact that an increase of dissolved particles in the fluid $P_{1}$ leads to more precipitation at the precipitate $P_{3}$, the microscopic precipitate region advances most quickly in $(49,24)^{\top}$ and most slowly in $(0,0)^{\top}$. Since the precipitates $P_{3}$ are completely surrounded by the fluids $P_{1}$, the precipitates advance uniformly in all spatial directions. At the end time the volume of the precipitate in $(49,24)^{\top}$ is around twelve times as much as the initial precipitate volume, whereas the volume of the precipitate in $(0,0)^{\top}$ is only around six times as much. Thus, the macroscopic field ${\overline{\phi_{3}}}^{\mathscr{P}}$ increases smoothly and monotonically on $\Omega_{h_{g}}$ from left to right and from bottom to top.

Figure 4.5 depicts the results of Test $S_{2}$. In contrast to Test $S_{1}$, the fluid $P_{2}$ is existent in $S_{2}$. Nevertheless, the same qualitative macroscopic results of the concentration $u$ and of the volume fraction $\bar{\phi}_{3} \mathscr{P}$ hold for the two tests. 


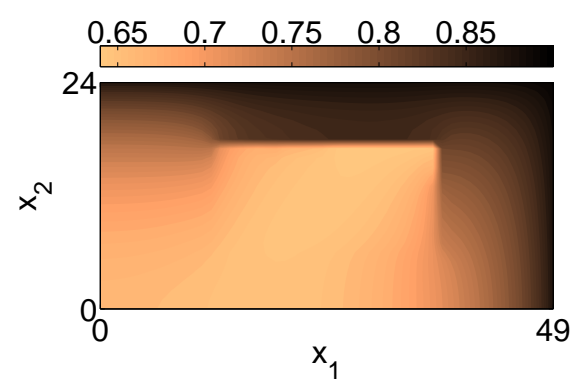

(a) Macroscopic $u$.

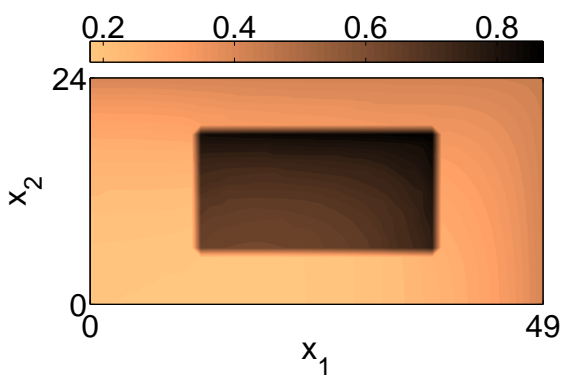

(b) Macroscopic ${\overline{\phi_{3}}}^{\mathscr{P}}$.

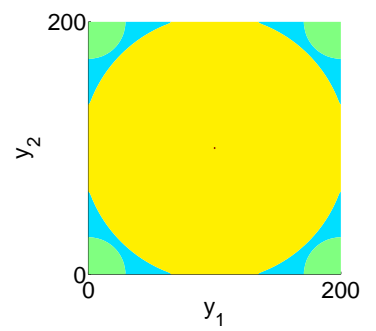

(c) Phases in $(36,18)^{\top}$.

FIG. 4.6: Results of Test $S_{3}$ in the end of $J_{\Delta^{n} t}(32)$.

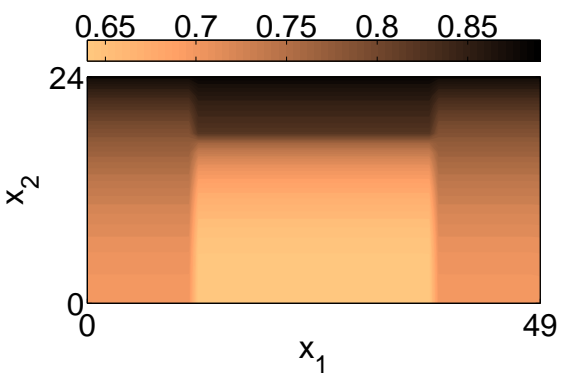

(a) Macroscopic $u$.

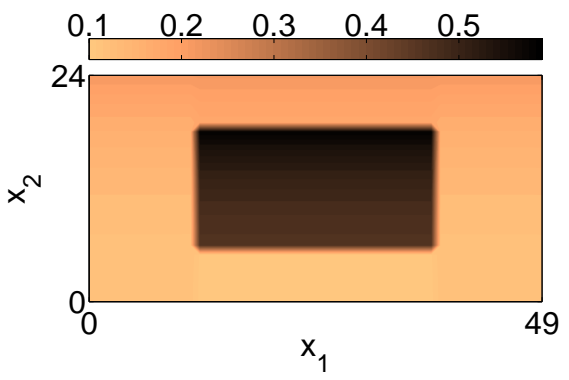

(b) Macroscopic ${\overline{\phi_{3}}}^{\mathscr{P}}$.

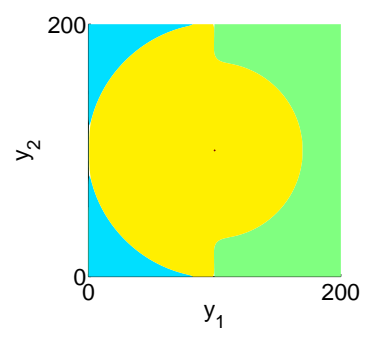

(c) Phases in $(36,18)^{\top}$.

FIG. 4.7: Results of Test $S_{4}$ in the end of $J_{\Delta^{n} t}(23)$.

\subsubsection{The Tests $S_{3}$ and $S_{4}$}

The results of Test $S_{3}$ are presented in Figure 4.6. For Test $S_{3}$ the macroscopic domain $\Omega_{h_{g}}$ is subdivided into the two sub-domains $\Omega_{h_{g}}^{(1)}=\Omega_{h_{g}} \backslash \Omega_{h_{g}}^{(2)}$ and $\Omega_{h_{g}}^{(2)}$ as depicted in Figure 4.1, where

$$
\Omega_{h_{g}}^{(2)}=\left\{x_{h_{g}} \in \Omega_{h_{g}} \mid x_{h_{g}} \in\left[12 h_{g}, 36 h_{g}\right] \times\left[6 h_{g}, 18 h_{g}\right]\right\} .
$$

The initial precipitate is of small radius $30 h_{\ell}$ in $\Omega_{h_{g}}^{(1)}$ and of large radius $70 h_{\ell}$ in $\Omega_{h_{g}}^{(2)}$. Therefore, $\overline{\phi_{3,0}} \mathscr{P}=0.0707$ in $\Omega_{h_{g}}^{(1)}$ and ${\overline{\phi_{3}}}^{\mathscr{P}}=0.3848$ in $\Omega_{h_{g}}^{(2)}$.

Since the initially constant concentration $u$ increases most quickly in the macroscopic node $(49,24)^{\top}$, due to the Neumann boundary condition (4.5), the precipitate region in $(49,24)^{\top}$ advances most quickly. Nevertheless, the largest precipitate is all the time located in $(36,18)^{\top}$. Because the volume fraction of the fluid $P_{1}$ in $\Omega_{h_{g}}^{(2)}$ is significantly smaller than in $\Omega_{h_{g}}^{(1)}$, the diffusion of the concentration $u$ is significantly weaker in $\Omega_{h_{g}}^{(2)}$ than in $\Omega_{h_{g}}^{(1)}$. The Figure 4.6a depicts an edge in the concentration field in $\Omega_{h_{g}}$ exactly where the Figure 4.6b depicts an edge in the volume fraction field ${\overline{\phi_{3}}}^{\mathscr{P}}$. An edge in ${\overline{\phi_{3}}}^{\mathscr{P}}$ corresponds to an edge in the volume fraction field ${\overline{\phi_{1}}}^{\mathscr{P}}$ of the fluid $P_{1}$. This is due to

$$
{\overline{\phi_{1}}}^{\mathscr{P}}=1-{\overline{\phi_{2}}}^{\mathscr{P}}-{\overline{\phi_{3}}}^{\mathscr{P}}
$$

and ${\overline{\phi_{2}}}^{\mathscr{P}}$ is almost constant in $J_{\Delta^{n} t}(32) \times \Omega_{h_{g}}$. This observation of stronger and weaker diffusion is of importance, because it matches the following real world phenomena. Interpreting clogging as the situation when the volume fraction of the fluid $P_{1}$ is very small and that actually the precipitate phase $P_{3}$ has grown up to the cell boundary, the dissolved particles in $P_{1}$ cannot diffuse on a macroscopic length scale.

Clogging is also observed in Test $S_{4}$, which is comparable to $S_{3}$ except for the initial configuration of the phases. For $S_{4}$ it is $\mathscr{I}_{1}$, instead of $\mathscr{I}_{2}$ for $S_{3}$ (Figure 4.1 depicts the different initial configurations). The left part of the nonprecipitate pore-space is occupied by the fluid $P_{1}$ and the remainder by $P_{2}$. Due to the fact that only the fluid $P_{1}$ contains 


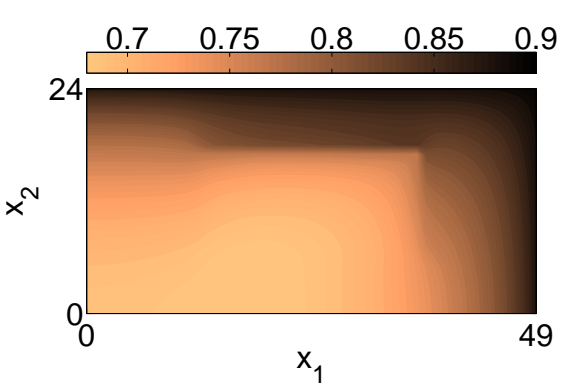

(a) Macroscopic $u$.

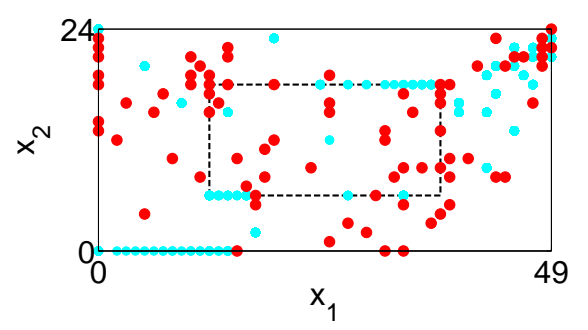

(d) Distribution of the active macroscopic nodes.

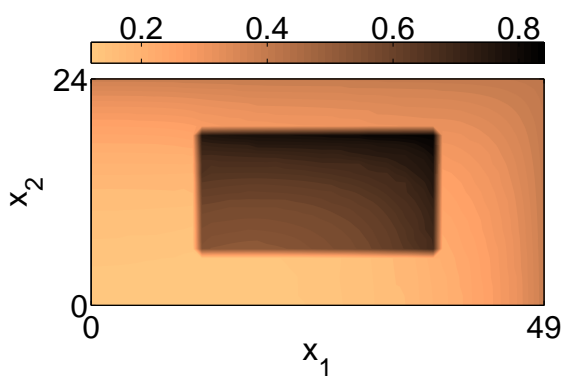

(b) Macroscopic ${\overline{\phi_{3}}}^{\mathscr{P}}$.

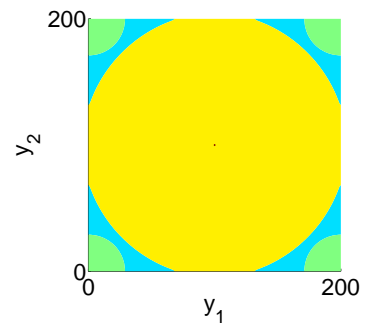

(c) Phases in $(36,18)^{\top}$.

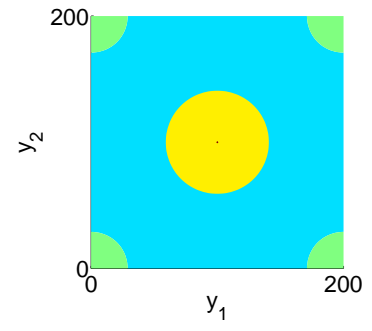

(e) Phases in $(0,0)^{\top}$.

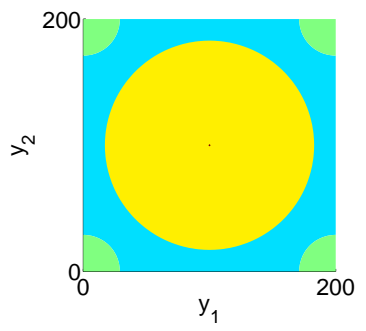

(f) Phases in $(12,6)^{\top}$.

FIG. 4.8: Results of Test $S_{5}$ in the end of $J_{\Delta^{n} t}(44)$. Figure (d) illustrates the distribution of the adaptive strategy's active nodes on the macroscopic domain: a dark dot denotes an active node in the end of $J_{\Delta^{n} t}(44)$, and a light dot signalizes that a node was active but became inactive in course of the simulation. The dashed line marks the sub-domain $\Omega_{h_{g}}^{(2)}$.

dissolved particles that can precipitate on a precipitate $P_{3}$, the precipitates advance only in contact with the fluid $P_{1}$. Besides precipitation, the evolution of a diffuse interface $\Gamma_{13}$ is influenced by its mean curvature, whereas the diffuse interfaces $\Gamma_{12}$ and $\Gamma_{23}$ evolve exclusively with mean curvature flow. Since the mean curvature of an interface $\Gamma_{12}$ is almost zero, the interfaces $\Gamma_{12}$ remain almost constant. Due to $\alpha=100$, the surface tensions $\sigma$ of the three phases are very small, that is $\sigma=\frac{3}{4 \alpha}=0.0075$ according to (2.83). Consequently, the evolution of the diffuse interface $\Gamma_{13}$ is dominated by the precipitation process. The diffuse interface $\Gamma_{23}$ is almost static. As a result, the change of the phases $P_{2}$ is not worth mentioning. It is important to note that the macroscopic concentration is influenced by an anisotropic diffusion, instead by an isotropic one in Test $S_{3}$. The diffusion is significantly stronger in vertical direction than in horizontal direction. This is a result of the configuration of the three phases in the microscopic pore-space. Unlike in the cases $\mathscr{I}_{0}$ and $\mathscr{I}_{2}$, it is not necessarily $w_{1}\left(y_{1}, y_{2}\right)=w_{2}\left(y_{2}, y_{1}\right)$ in $\mathscr{P}_{h_{\ell}}$. Consequently, the diffusion tensor $\overline{K\left(\phi_{1}+\delta, w_{1}, w_{2}\right)} \mathscr{P}$ is anisotropic.

\subsubsection{The Test $S_{5}$}

Finally, Figure 4.8 presents the results of Test $S_{5}$. Test $S_{5}$ differs from $S_{3}$ only in the parameter $u_{0}$. In $S_{5}$ the initial concentration $u_{0}=0.35$ of dissolved particles in $P_{1}$ is below the threshold $c_{u, 1}=0.5$ of the rate-function $f$ defined in (2.5). Consequently, the precipitates recede in the beginning of $J_{\Delta^{n} t}(44)$, that is precipitate particles of $P_{3}$ dissolve in the fluid $P_{1}$. Due to the dissolution and the transport of particles across the boundary of $\Omega_{h_{g}}$ the concentration $u$ in $P_{1}$ increases. From the moment the concentration exceeds the threshold, the precipitate regions advance again as a result of precipitation. The resulting edges in the concentration field and in the volume fraction field ${\overline{\phi_{3}}}^{\mathscr{P}}$ in $\Omega_{h_{g}}$ are not as distinct as in $S_{3}$. This is due to the initial retreat of the precipitates in $S_{5}$ and the resulting larger volume fractions ${\overline{\phi_{1}}}^{\mathscr{P}}$.

Figure 4.8 shows also the distribution of the active macroscopic nodes of the adaptive strategy. Only the microscopic problems in active macroscopic nodes are solved. Most of them are located in the areas of $\Omega_{h_{g}}$ with steep slopes in the concentration field, since steep slopes yield (in general) outstanding concentration evolutions. The evolutions of the concentrations in the areas of $\Omega_{h_{g}}$ with moderate slopes are usually very similar. On average only around $7 \%$ of 
the 1,250 macroscopic nodes are active in $J_{\Delta^{n} t}(44)$.

\section{Conclusion and outlook}

The combination of, firstly, three pore-scale Allen-Cahn type equations modeling the evolution of three phase-fields for three sub-domains occupied by three different species, secondly, a pore-scale Diffuse Domain Approximation in the sense of Li et al. (2009), and thirdly, the application of a homogenization technique to the pore-scale model led us to a two-scale tri-phase phase-field model for precipitation in a porous medium that allows the use of an efficient numerical method.

We expect that our approach can be generalized in several directions. The extension of the model to a situation with more than three species appears to be straightforward. Only the construction of the interpolation function $\bar{g}=\bar{g}(\Phi)$ is intricate since more trace conditions must be considered. The inclusion of fluid flow into the model is more involved. Since Allen-Cahn equations are not mass conservative, it is possible that convection effects in the pore-space lead to the full absorption of a fluid by the precipitate substance. On the contrary, Cahn-Hilliard equations ensure the conservation of mass. Therefore a natural next step would be the use of incompressible Navier-Stokes-Cahn-Hilliard models like in Feng et al. (2007); Lowengrub \& Truskinovsky (1998) (see Boyer et al. (2010) for the three-phase case) as pore scale models. However, it is fundamental to the DDA method that the phase-fields stay within the interval $[0,1]$. Besides the inclusion of full fluid flow into the problem, an analysis for the pore-scale as well as for the upscaled phase-field model is a major part of future research.

The presented numerical results document a strong inter-scale interdependence of the evolutions of the Darcy-scale concentration of dissolved precipitate in the water phase and of the pore-scale domain occupied by the water. The larger the water fraction on the pore domain the stronger the Darcy-scale diffusion. In particular, the Darcy-scale diffusion tensor is not necessarily isotropic, since it depends on the shape of the pore-scale water domain. Furthermore, the larger the Darcy-scale concentration the faster the growth of the pore-scale precipitate.

\section{Acknowledgement}

The authors are members of the International Research Training Group NUPUS funded by the German Research Foundation DFG (GRK 1398), the Netherlands Organisation for Scientific Research NWO (DN 81-754) and by the Research Council of Norway (215627). This support is gratefully acknowledged. Also many thanks to Michael Kutter, Tycho van Noorden, Iryna Rybak, Gorkem Simsek and Kris van der Zee for stimulating discussions.

\section{REFERENCES}

Boyer, F. \& Lapuerta, C. (2006) Study Of A Three Component Cahn-Hilliard Flow Model. ESAIM: M2AN, 40(4), $653-687$.

Boyer, F., Lapuerta, C., Minjeaud, S., Piar, B. \& Quintard, M. (2010) Cahn-Hilliard/Navier-Stokes Model for the Simulation of Three-Phase Flow. Transp Porous Med, 82, 463-483.

Bronsard, L. \& Reitich, F. (1993) On Three-Phase Boundary Motion and the Singular Limit of a Vector-Valued Ginzburg-Landau Equation. Arch. Rational Mech. Anal., 124, 355-379.

Caginalp, G. (1986) An analysis of a phase field model of a free boundary. Arch. Ration. Mech. Anal., 92, $205-245$.

Caginalp, G. \& Fife, P. (1988) Dynamics of Layered Interfaces Arising from Phase Boundaries. SIAM J. Appl. Math., 48(3), 506518.

Eck, C. \& Emmerich, H. (2009) A two-scale model for liquid-phase epitaxy. Math. Methods Appl. Sci., 32(1), $12-40$.

Eck, C., Kutter, M., Sändig, A.-M. \& Rohde, C. (2013) A two scale model for liquid phase epitaxy with elasticity: an iterative procedure. ZAMM Z. Angew. Math. Mech., 93(10-11), 745-761.

Evans, L. (2010) Partial Differential Equations. Graduate studies in mathematics. American Mathematical Society.

Feng, X., He, Y. \& Liu, C. (2007) Analysis of finite element approximations of a phase field model for two-phase fluids. 76(258), 539-571.

Hornung, U., editor (1997) Homogenization and Porous Media. Springer, New York.

Knabner, P., Van Duijn, C. \& Hengst, S. (1995) An analysis of crystal dissolution fronts in flows through porous media. Part 1: Compatible boundary conditions. Adv. Water Resour., 18(3), 171-185.

Kockelkoren, J., Levine, H. \& Rappel, W.-J. (2003) Computational approach for modeling intra- and extracellular dynamics. Phys. Rev. $E, \mathbf{6 8}, 037702$.

Kumar, K., Noorden, T. L. v. \& Pop, I. S. (2011) Effective dispersion equations for reactive flows involving free boundaries at the microscale. Multiscale Model. Simul., 9(1), 29-58. 
Kumar, K., T., W. \& Wheeler, M. (2013) Reactive flow in a thin channel and reaction-induced boundary movement in a monolithic ALE framework. SIAM J. Sci. Comp., 6, B1235-B1266.

Li, X., Lowengrub, J., Rätz, A. \& Voigt, A. (2009) Solving PDEs In Complex Geometries: A Diffuse Domain Approach. Commun. Math. Sci., 7, 81-107.

Lowengrub, J. \& Truskinovsky, L. (1998) Quasi-incompressible Cahn-Hilliard fluids and topological transitions. Proc. R. Soc. Lond. A, 454(1978), 2617-2654.

Meier, S. (2008) A homogenisation-based two-scale model for reactive transport in media with evolving microstructure. $C$. $R$. Mecanique, 336, 623-628.

Muntean, A. \& Noorden, T. L. v. (2013) Corrector estimates for the homogenization of a locally periodic medium with areas of low and high diffusivity. European J. Appl. Math., 24(5), 657-677.

Noorden, T. L. v. (2009a) Crystal precipitation and dissolution in a porous medium: effective equations and numerical experiments. Multiscale Model. Simul., 7(3), 1220-1236.

Noorden, T. L. v. (2009b) Crystal precipitation and dissolution in a thin strip. European J. Appl. Math., 20(1), 69-91.

Noorden, T. L. v. \& Eck, C. (2011) Phase field approximation of a kinetic moving-boundary problem modelling dissolution and precipitation. Interface. Free Bound., 13, 29-55.

Noorden, T. L. v. \& Muntean, A. (2011) Homogenisation of a locally periodic medium with areas of low and high diffusivity. European J. Appl. Math., 22(5), 493-516.

Noorden, T. L. v. \& Pop, I. S. (2008) A Stefan problem modelling crystal dissolution and precepitation. IMA J. Appl. Math., 73(2), 393-411.

Peter, M. A. (2009) Coupled reaction-diffusion processes inducing an evolution of the microstructure: analysis and homogenization. Nonlinear Anal., 70(2), 806-821.

Ray, N., van Noorden, T., Radu, F., Friess, W. \& Knabner, P. (2013) Drug release from collagen matrices including an evolving microstructure. ZAMM Z. Angew. Math. Mech., 93, 811-822.

Redeker, M. \& Eck, C. (2013) A fast and accurate adaptive solution strategy for two-scale models with continuous inter-scale dependencies. J. Comput. Phys., 240, 268-283.

Redeker, M. \& Haasdonk, B. (2014) A POD-EIM reduced two-scale model for crystal growth. Adv. Comput. Math., pages 1-27.

Renardy, M. \& Rogers, R. (2004) An Introduction to Partial Differential Equations. Springer.

Roubicek, T. (2005) Nonlinear partial differential equations with applications. Springer. 